\title{
Patterns in the effects of infectious diseases on population growth
}

\author{
O. Diekmann ${ }^{1,2}$ and M. Kretzschmar**** \\ 1 Centrum voor Wiskunde en Informatica, Kruislaan 413, NL-1098 SJ Amsterdam \\ 2 Institute for Theoretical Biology, Kaiserstraat 63, NL-2311 GP Leiden
}

Received December 20, 1989; received in revised form September 14, 1990

\begin{abstract}
An infectious disease may reduce or even stop the exponential growth of a population. We consider two very simple models for microparasitic and macroparasitic diseases, respectively, and study how the effect depends on a contact parameter $\kappa$. The results are presented as bifurcation diagrams involving several threshold values of $\kappa$. The precise form of the bifurcation diagram depends critically on a second parameter $\xi$, measuring the influence of the disease on the fertility of the hosts. A striking outcome of the analysis is that for certain ranges of parameter values bistable behaviour occurs: either the population grows exponentially or it oscillates periodically with large amplitude.
\end{abstract}

Key words: Epidemic - Population regulation - Threshold values for contact parameter - Bistable behaviour - Oscillations

\section{Introduction}

One of the aims of mathematical modelling in population dynamics is to find a causal relationship between phenomena and the underlying mechanisms. We want to understand how mechanisms, which act in and between the individuals of a population, determine size and structure of the population as a whole. This aim justifies to some extent the study of simple and consequently unrealistic models, where "unrealistic" means that they lack predictive value. Simple and mathematically tractable models in terms of a few variables allow us to develop some idea of the way in which properties of individuals (as incorporated in the model structure and summarized in parameters) influence the dynamical behaviour of the system. The investigation of the similarities and differences in dynamical behaviour of models which are identical in all but one structural component may yield insight in the influence of the biological mechanism described by this particular component.

In this paper we study the dynamics of two simple epidemic models. One of them is a model for microparasitic diseases of the $S-I$ type, the other one is a

* Present address: Department of Statistics and Modelling Science, University of Strathclyde, Livingstone Tower, Glasgow G1 1XH Scotland, UK

** The work of this author was supported by a grant of the Deutsche Forschungsgemeinschaft (DFG) 
model for a host-parasite system. We consider a population which grows exponentially in the absence of the disease. We are interested in the regulation problem: how does the disease affect the growth rate of its host population? So we look at a time scale at which the demographic processes as well as the disease transmission are important and we want to know what kind of dynamical behaviour can result from their interaction. Of course the answer depends on many parameters. Our strategy will be to look for patterns of changes in dynamical behaviour as a contact rate parameter, which measures the infective strength, is increased. Our results will, in the spirit of May and Anderson [1,16] and Busenberg and van den Driesche [5], identify various thresholds for this contact rate parameter. Moreover, we will find that the pattern of changes of dynamical behaviour for diseases which have a strong influence on fertility is different from that for diseases which have no or only a minor influence on fertility. Among other things this clarifies in a much simpler context some observations of Kretzschmar [13] about subcritical bifurcations and the possibility of bistable behaviour.

For populations which possibly grow beyond every bound the use of strict mass action kinetics is questionable. Some authors dealing with models for STD's ( sexually transmitted diseases) or worm diseases have introduced homogeneous models, i.e. models in which the total population size is but a scaling variable (see $[7,8,9])$. Such models allow for exponential solutions, as we will see in detail below. Of course homogeneous contact rates are debatable at low population sizes and hence for the exponential solutions to be meaningful it is required that the exponent is positive. Another class of models involves a contact rate which is like the strict law of mass action at low population densities, but essentially homogeneous for high population densities. Such a contact rate can be introduced as a phenomenological description (see Dietz [6]) or as resulting from application of a time scale argument to a free living infective stage ([17], also see Subsect. 2.2 below). The models now allow the existence of endemic steady states. One of our aims in this paper is to demonstrate how exponential solutions with positive exponents occur as asymptotic solutions of the second class of models and how they connect to the steady states when the contact rate parameter varies.

We will first introduce the models and explain the biological meaning of the parameters. Next we will summarize the results for the two models in biological terms. Finally we are going to give a unified formulation for the two systems of model equations which we then analyse mathematically. We conclude with some remarks about the biological interpretation of the results.

\section{Modelling microparasitic and macroparasitic diseases}

The distinction between micro- and macroparasites was introduced by Anderson and May in two articles published in Nature in $1972[2,17]$. They classify diseases that are caused by a virus or by bacteria as microparasitic and diseases caused by helminths or arthropods as macroparasitic diseases. Epidemiologically the main difference is that in microparasitic diseases possible reinfection of an already infected individual plays no role in the disease dynamics, while in macroparasitic diseases the number of reinfections, or, more precisely, the number of parasites per host, has to be taken into account when modelling the disease. 
The main common features of the models introduced here are

1. Both models will allow for an effect of the disease on the fertility of the infective individuals.

2. The infection rate in both models will be an asymptotically homogeneous function, i.e. for large population sizes the infection rate will be approximately proportional to the fraction of infectives in the total population (microparasitic diseases) or proportional to the mean parasite load of the host population (macroparasitic diseases).

\subsection{Model I: Microparasitic diseases}

The model we introduce for microparasitic diseases is a $S$ - $I$ model, i.e. the population is divided into the class of infectives $I$ and the class of susceptibles $S$. We take the following parameters into account:

$\beta$ : per capita natural birth rate

$\mu$ : per capita natural death rate

$\alpha$ : additional mortality rate caused by the disease

$\xi$ : parameter describing the reduction of fertility of an infected individual due to the disease $(0 \leqslant \xi \leqslant 1)$

$\kappa$ : contact rate between infectives and susceptibles.

$\varphi$ : infection rate (to be specified).

The reduction of fertility in the population due to the disease is incorporated into the model by describing its effect on pairs within the population, i.e. by including a very crude description of pair formation. (For a discussion of pair formation models see $[4,7,18]$.) A pair can be formed by two susceptibles, a susceptible and an infective, or two infectives. We assume random mixing. The rate of producing offspring is then reduced by the factor $\xi$ or $\xi^{2}$, respectively, depending on whether one or both partners of a pair are infective. Thus the presence of infectives also reduces the fertility of susceptibles.

The model is represented by the system of ordinary differential equations

$$
\begin{aligned}
& \frac{d S}{d t}=\beta \frac{S^{2}+2 \xi I S+\xi^{2} I^{2}}{I+S}-\mu S-\varphi S, \\
& \frac{d I}{d t}=\varphi S-\mu I-\alpha I .
\end{aligned}
$$

The infection rate $\varphi$ is described by a function which displays a saturation effect accounting for the fact that the number of contacts an individual can have with other individuals reaches some finite maximal value due to the spatial or social distribution of the population and/or limitation of time (cf. the derivation of a Holling type II functional response in predator-prey models). More precisely, we take $\varphi$ as

$$
\varphi=\frac{\kappa I}{c+S+I}
$$


where $c$ is a constant. Thus model $\mathrm{I}$ is given by the system

$$
\begin{aligned}
& \frac{d S}{d t}=\beta \frac{S^{2}+2 \xi I S+\xi^{2} I^{2}}{I+S}-\mu S-\frac{\kappa I S}{c+S+I}, \\
& \frac{d I}{d t}=\frac{\kappa I S}{c+S+I}-\mu I-\alpha I .
\end{aligned}
$$

\subsection{Model II: Macroparasitic diseases}

The model for macroparasitic diseases that we want to discuss is based on a model that was introduced by Anderson and May in 1978 [1, 16]. It describes the dynamics of a host population $N$ and a parasite population $P$. In order to determine how the parasite load of the host influences the dynamics of the populations one starts out with a more complicated model which takes "wormload" structure into account and then simplifies the system by making an assumption about the distribution of parasites on the host population. Anderson and May showed that data concerning number of parasites per host can be fitted very well with a negative binomial distribution. Therefore in the model one assumes that the parasites are distributed on the hosts according to a negative binomial distribution with the mean given by the mean parasite load $P / N$ and a parameter $k$ describing the so-called "clumping". A small $k$ indicates high clumping, i.e. few hosts carry a large part of the parasites, while a large part of all hosts have only very few parasites. For $k \rightarrow \infty$ the distribution approaches a Poisson distribution, i.e. the parasites are randomly distributed over the host population. In the original model by Anderson and May the parasites only influenced the mortality of the host, not its fertility. In a variant Anderson and May [16] also discussed influence on fertility, but used an approach that is different from the one introduced here, which follows Dietz and Hadeler [8,9] and Kretzschmar [13]. The following parameters occur in the model:

$\beta$ : per capita natural birth rate of hosts

$\mu$ : per capita natural death rate of hosts

$\alpha$ : additional mortality rate caused by one parasite

$\xi$ : parameter describing the reduction of fertility of an infected individual due to one parasite $(0 \leqslant \xi \leqslant 1)$

$\kappa$ : contact rate between hosts and infective stages of the parasites

$\varphi$ : infection rate (to be specified)

$\sigma:$ death rate of parasites

$k$ : clumping parameter.

The model is derived from the infinite system of differential equations which describes an immigration-death process of parasites on the hosts. If we denote for $i \in \mathbb{N}_{0}$ by $n_{i}(t)$ the number of hosts which carry $i$ parasites, then the following system of equations describes birth and death of hosts and immigration and death of parasites:

$$
\begin{gathered}
\frac{d}{d t} n_{0}=-(\mu+\varphi) n_{0}+\sigma n_{1}+\beta \sum_{i=0}^{\infty} n_{i} \xi^{i}, \\
\frac{d}{d t} n_{i}=-(\mu+\varphi+i(\alpha+\sigma)) n_{i}+\sigma(i+1) n_{i+1}+\varphi n_{i-1}, \quad i \geqslant 1 .
\end{gathered}
$$


Now we define

$$
N(t):=\sum_{i=0}^{\infty} n_{i} \quad \text { and } \quad P(t):=\sum_{i=0}^{\infty} i n_{i}
$$

and derive the following equations from (2.4)

$$
\begin{aligned}
& \frac{d}{d t} N=-\mu N-\alpha P+\beta \sum_{i=0}^{\infty} n_{i} \xi^{i}, \\
& \frac{d}{d t} P=-(\mu+\sigma) P+\varphi N-\alpha \sum_{i=0}^{\infty} i^{2} n_{i} .
\end{aligned}
$$

In order to express the sums in Eqs. (2.6) in terms of $N$ and $P$ we assume that the parasites are distributed on the host population according to a negative binomial distribution with mean $P / N$ and a "clumping parameter" $k$. We use the generating function of the negative binomial distribution to deduce that

$$
\begin{gathered}
\sum_{i=0}^{\infty} n_{i} \xi^{i}=N\left(\frac{k N}{(1-\xi) P+k N}\right)^{k}, \\
\sum_{i=0}^{\infty} i^{2} n_{i}=N\left(\frac{P}{N}+\left(\frac{k+1}{k}\right)\left(\frac{P}{N}\right)^{2}\right) .
\end{gathered}
$$

This leads to a closed system of equations for $N$ and $P$ :

$$
\begin{aligned}
& \frac{d}{d t} N=-\mu N-\alpha P+\beta N\left(\frac{k N}{(1-\xi) P+k N}\right)^{k}, \\
& \frac{d}{d t} P=-(\mu+\sigma) P+\varphi N-\alpha N\left(\frac{P}{N}+\left(\frac{k+1}{k}\right)\left(\frac{P}{N}\right)^{2}\right) .
\end{aligned}
$$

We now also want to express the infection rate $\varphi$ in terms of $N$ and $P$. In order to do that we have to take the indirect transmission of parasites into account. If we denote by $W(t)$ the number of free living larvae of the parasite, which are infectious for the host, we can describe the dynamics of $W(t)$ by the equation

$$
\frac{d}{d t} W=\kappa P-\varphi N-\gamma W
$$

This means that the production of larvae is proportional to the number of parasites, the larvae are taken up by the host with rate $\varphi$ and die with rate $\gamma$. Furthermore we assume that $\varphi=\theta W$ with some constant $\theta$. Now we use a time scale argument. We know that for many parasitic diseases the life span of the larvae is very short compared with the life spans of hosts and adult parasites. This means that on the time scale that we are interested in the larvae population is always practically at equilibrium. Consequently we put

$$
W=\frac{\kappa P}{\gamma+\theta N} \text {. }
$$

Defining $c:=\gamma / \theta$ we arrive at

$$
\varphi=\frac{\kappa P}{c+N}
$$


and the model is now given by the system of equations

$$
\begin{aligned}
& \frac{d N}{d t}=-\alpha P-\mu N+\beta N\left(\frac{k N}{(1-\xi) P+k N}\right)^{k}, \\
& \frac{d P}{d t}=P\left(\frac{\kappa N}{c+N}-(\mu+\sigma+\alpha)-\alpha \frac{(k+1)}{k} \frac{P}{N}\right) .
\end{aligned}
$$

For $\xi=1$ this reduces to the model that was investigated by Anderson and May.

The wormload models of May and Anderson and of Dietz and Hadeler differ in several respects. May and Anderson have a saturating infection rate, they use the "negative binomial assumption" and they pay hardly any attention to effects on fertility (in fact their approach to include effects on fertility in an "additive" way may lead to the absurdity of a negative birth rate). Dietz and Hadeler take a homogeneous infection rate, they study the full model, i.e. the infinite system of differential equations, even including age structure of the host population, and they consider "multiplicative" effects on fertility. One of the aims of our exercise with the hybrid version (2.10) is to be able to disentangle how differences in modelling and differences in qualitative behaviour are related to each other.

In this paper we especially concentrate on the contact rate $\kappa$ and on the parameter $\xi$. More precisely, we investigate the bifurcation diagram with $\kappa$ as a parameter and how this bifurcation diagram depends on $\xi$.

\section{Patterns of dynamical behaviour}

In this section we summarize the results for models I and II, respectively, and give a biological interpretation of the results. The mathematical proofs are delayed to Sect. 4. Throughout this paper we assume that $\beta>\mu$, which means that the population will grow exponentially in the absence of the disease.

\subsection{No influence on fertility}

We first discuss the dynamics for the case $\xi=1$, i.e. for the case that the disease has no influence on the fertility of the infectives/hosts.

For both models the qualitative behaviour of the solutions depends on the value of the parameter $\kappa$, which can be interpreted as a parameter describing the contact rate between infectives and susceptibles, or between hosts and parasites, respectively. We can compute three threshold values $\kappa_{0}, \kappa_{1}, \kappa_{2}$ for which the qualitative behaviour changes.

1. For $0 \leqslant \kappa<\kappa_{0}$ the disease dies out for $t \rightarrow \infty$ and the population of susceptibles/hosts eventually grows exponentially with growth rate $\beta-\mu$.

2. For $\kappa_{0}<\kappa<\kappa_{1}$ both the populations of susceptibles/hosts and infectives/ parasites eventually grow exponentially, the susceptibles/hosts with the rate $\beta-\mu$, the infectives/parasites with a smaller rate. Here we have a "dilution effect": although the disease does not die out, the fraction of infectives in the total population (model I) and the mean parasite load of the hosts (model II) tend to 0 . 
3. For $\kappa_{1}<\kappa<\kappa_{2}$ both populations eventually grow exponentially with the same rate, which is now smaller than $\beta-\mu$. This means that in this case the disease has a long term influence on the demographic process of the population.

4. For $\kappa>\kappa_{2}$ there exists an asymptotically stable equilibrium. In other words, the disease has the ability to regulate the population to a steady state, in which the disease is endemic. For model I this threshold only exists, if the additional condition $\beta-\mu<\alpha$ is fulfilled.

For model I the threshold values can be calculated as

$$
\begin{aligned}
& \kappa_{0}=\mu+\alpha, \\
& \kappa_{1}=\beta+\alpha, \\
& \kappa_{2}=\frac{\alpha(\mu+\alpha)}{\mu+\alpha-\beta} .
\end{aligned}
$$

For model II we get the threshold values:

$$
\begin{aligned}
& \kappa_{0}=\mu+\sigma+\alpha, \\
& \kappa_{1}=\beta+\sigma+\alpha, \\
& \kappa_{2}=\mu+\sigma+\alpha+(\beta-\mu)\left(\frac{k+1}{k}\right) .
\end{aligned}
$$

One can easily calculate and interpret these critical values on the basis of the following formal arguments. (We concentrate on model I.)

Assuming that $S \rightarrow \infty$ and $I / S \rightarrow 0$ for $t \rightarrow \infty$ we find that asymptotically the second equation of (2.3) reduces to

$$
\frac{d I}{d t} \approx(\kappa-\mu-\alpha) I
$$

Hence $I$ will grow if and only if $\kappa>\kappa_{0}=\mu+\alpha$, where $\kappa$ is the asymptotic per capita "birth" rate of $I$ and $\mu+\alpha$ the death rate. Under the same conditions the first equation of (2.3) reduces to

$$
\frac{d S}{d t} \approx(\beta-\mu) S
$$

so $S$ will grow asymptotically with rate $\beta-\mu$. These findings are consistent with the assumption $I / S \rightarrow 0$ provided

$$
\kappa-\mu-\alpha<\beta-\mu \text {, i.e. } \kappa<\kappa_{1}=\beta+\alpha .
$$

For larger values of $\kappa$ we may make the ansatz that $I / S \rightarrow \theta>0$, while still assuming that $S \rightarrow \infty$. Then (2.3) is asymptotically decoupled:

$$
\begin{aligned}
\frac{d S}{d t} & =\left(\beta(1+\theta)-\mu-\frac{\kappa \theta}{1+\theta}\right) S, \\
\frac{d I}{d t} & =\left(\frac{\kappa}{1+\theta}-\mu-\alpha\right) I .
\end{aligned}
$$

Consistency with $I / S \rightarrow \theta$ requires that

$$
\beta(1+\theta)-\mu-\frac{\kappa \theta}{1+\theta}=\frac{\kappa}{1+\theta}-\mu-\alpha
$$


or, in other words, that

$$
\theta=\frac{\kappa-\alpha-\beta}{\beta}
$$

The common growth rate of $S$ and $I$ then equals

$$
\frac{\kappa \beta}{\kappa-\alpha}-\mu-\alpha
$$

Consistency with $S \rightarrow \infty$ requires this to be positive, or

$$
\kappa<\kappa_{2}=\frac{\alpha(\mu+\alpha)}{\mu+\alpha-\beta}
$$

whenever $\beta<\mu+\alpha$.

The calculations for model II follow the same pattern, only the details differ.

\subsection{Influence on fertility}

If we allow for the disease to have a strong negative influence on the fertility of the infective individuals, the spectrum of possibilities for the dynamic behaviour of the solutions becomes richer and the pattern of changes in qualitative behaviour with increasing $\kappa$ is different. Both for models I and II it will be shown that there exist values $\xi_{T}$ and $\xi_{p}$ (the index $T$ refers to "turning point" and the index $p$ to "periodic solution"), so that

- for $\xi>\xi_{T}$ the pattern is the same as described in Subsect. 3.1,

- for $\xi_{p}<\xi<\xi_{T}$ there exists an additional threshold value $\kappa_{T}$ marking the beginning of a region of $\kappa$-values for which there is bistability,

- for $0<\xi<\xi_{p}$ there exists, in addition, a threshold value $\kappa_{p}$ marking the beginning of a region of $\kappa$-values for which stable periodic solutions occur.

More precisely, for $\xi<\xi_{T}$ the qualitative behaviour of solutions depends on the parameter $\kappa$ as follows:

1. For $0 \leqslant \kappa<\min \left(\kappa_{0}, \kappa_{T}\right)$ we have the same as in Subsect. 3.1, case 1 .

2. When $\kappa_{T}>\kappa_{0}$ we have for $\kappa_{0}<\kappa<\kappa_{T}$ the same situation as in Subsect. 3.1, case 2 .

3. When $\kappa_{T}<\kappa_{0}$ then for $\kappa_{T}<\kappa<\kappa_{0}$ either the disease dies out or both (sub) populations grow with the same reduced growth rate. It depends on the initial condition which of the two possibilities actually occurs.

4. For $\max \left(\kappa_{0}, \kappa_{T}\right)<\kappa<\kappa_{1}$ the long term dynamical behaviour depends on the initial conditions. For certain regions of initial conditions we again have the "dilution effect": exponential growth of both (sub)populations, but the fraction of infectives/mean parasite load goes to 0 . For other regions of initial conditions the long term behaviour is exponential growth with the same reduced rate for both (sub)populations.

5. For $\kappa>\kappa_{2}$ there exists an equilibrium solution, which is a stable node or spiral for all $\kappa>\kappa_{2}$ in the case $\xi>\xi_{p}$, while it is unstable for all $\kappa>\kappa_{2}$ in the case $\xi<\xi_{p}$. For $\xi<\xi_{p}$, i.e. if the equilibrium is unstable, there exists a stable limit cycle for $\kappa>\kappa_{p}$ (the index $p$ stands for "periodic"). This means that the population sizes of susceptibles/hosts and infectives/parasites oscillate in time. In 
numerical solutions we could observe an extreme "outbreak" behaviour, i.e. the number of infectives/parasites is almost 0 until the susceptible/host population has reached some threshold size. Then a huge outburst of the infective/parasite population occurs, which quickly breaks down again.

6. In contrast to Subsect. 3.1 we do not necessarily have $\kappa_{1}<\kappa_{2}$. If $\kappa_{2}<\kappa_{1}$ we have coexistence of a stable equilibrium or a periodic solution and the "dilution effect" situation. It again depends on the initial condition, whether the disease is able to regulate the population to a steady or periodic state in which it is endemic, or whether it is outgrown by the rapid instream of susceptibles.

These results are summarized in the bifurcation diagrams in Subsect. 4.4.

The thresholds $\kappa_{0}$ and $\kappa_{1}$ for both models are the same as in Subsect. 3.1. Furthermore we get the following thresholds:

For model I:

$$
\kappa_{2}=\frac{\mu+\alpha}{1-d_{1}}
$$

with (we write $\eta=1-\xi$ )

$$
d_{1}=\frac{1}{2 \eta^{2}}\left(2 \eta+\frac{\alpha}{\beta}-\sqrt{\left(2 \eta+\frac{\alpha}{\beta}\right)^{2}-4 \eta^{2}\left(1-\frac{\mu}{\beta}\right)}\right)
$$

The threshold $\kappa_{T}$ for model $\mathrm{I}$ is given by

$$
\kappa_{T}=\alpha+4 \beta \xi(1-\xi) \text {. }
$$

Note that $\kappa_{T} \rightarrow \alpha$ for $\xi \rightarrow 0$, so $\kappa_{T}$ can indeed be smaller than $\kappa_{0}$. The thresholds for $\xi$ are given by

$$
\xi_{T}=\frac{1}{2} \text { and } \xi_{p}=1-\eta_{p}
$$

where $\eta_{p}$ is the unique solution in $\left[\frac{1}{2}, 1\right]$ of the cubic equation

$$
4 \beta \eta^{3}-8 \beta \eta^{2}+(4 \beta-2 \alpha-\mu) \eta+\alpha=0 .
$$

It is not possible to calculate $\kappa_{p}$ explicitly.

For model II we get the following thresholds:

$$
\kappa_{2}=\mu+\sigma+\alpha+d_{2} \alpha\left(\frac{k+1}{k}\right),
$$

with a positive constant $d_{2}$, which again depends on the parameters of the model (except $\kappa$ ). In this case, however, we cannot write down an explicit expression for $d_{2}$. Similarly $\kappa_{T}$ and $\kappa_{p}$ cannot be calculated explicitly. The threshold $\xi_{T}$ is given by

$$
\xi_{T}=1-\frac{\alpha}{\beta k},
$$

while $\xi_{p}$ cannot be calculated explicitly.

Again we can derive and interpret the critical parameter values by performing formal calculations.

When we assume that $I / S \rightarrow \theta$ and $S \rightarrow \infty$ we find, in model I, that the asymptotic growth rates of $S$ and $I$ are, respectively,

$$
\beta f(\theta, \xi)-\mu-\frac{\kappa \theta}{1+\theta},
$$


and

$$
\frac{\kappa}{1+\theta}-\mu-\alpha
$$

where

$$
f(\theta, \xi):=\frac{(1+\theta \xi)^{2}}{1+\theta}
$$

describes the apparent per capita birth rate of susceptibles. Increasing $\theta$ now has two opposite effects: more individuals contribute to reproduction, which makes $f$ larger, but the average per capita birth rate of all individuals decreases when $\xi<1$, which makes $f$ smaller. It depends on $\xi$, which effect is more important. Since

$$
\frac{\partial f}{\partial \theta}(\theta, \xi)=(2 \xi-1+\theta \xi) \frac{1+\theta \xi}{(1+\theta)^{2}}
$$

we conclude that

$$
\frac{\partial f}{\partial \theta}>0 \text { for } \xi \geqslant \frac{1}{2} \text { and } \theta>0,
$$

whereas for $\xi<\frac{1}{2}$ we have

$$
\frac{\partial f}{\partial \theta}<0 \text { for } \theta<\frac{1-2 \xi}{\xi},
$$

and

$$
\frac{\partial f}{\partial \theta}>0 \text { for } \theta>\frac{1-2 \xi}{\xi} .
$$

Define $\xi_{T}=\frac{1}{2}$. The minimum value of $f$ as a function of $\theta$ is

$$
\begin{gathered}
f(0, \xi)=1 \text { for } \xi \geqslant \xi_{T}, \\
f\left(\frac{1-2 \xi}{\xi}, \xi\right)=4 \xi(1-\xi) \text { for } \xi<\xi_{T} .
\end{gathered}
$$

Equality of the growth rates of $S$ and $I$ requires

$$
\kappa=\alpha+\beta f(\theta, \xi)
$$

Define

$$
\kappa_{1}=\alpha+\beta f(0, \xi)=\alpha+\beta
$$

and, for $\xi<\xi_{T}$,

$$
\kappa_{T}=\alpha+\beta \min f=\alpha+4 \beta \xi(1-\xi) .
$$

We conclude that for $\xi<\xi_{T}$ and $\kappa_{T}<\kappa<\kappa_{1}$ two values of $\theta$ lead to consistency (see Fig. 1).

Rather than solving for $\theta$, given $\kappa$ and the other parameters, we may use $\theta$ as a parameter which determines $\kappa$. Along the curve $\kappa=\alpha+\beta f(\theta, \xi)$ the common growth rate of $S$ and $I$ is given by

$$
\frac{\alpha+\beta f(\theta, \xi)}{1+\theta}-\mu-\alpha
$$




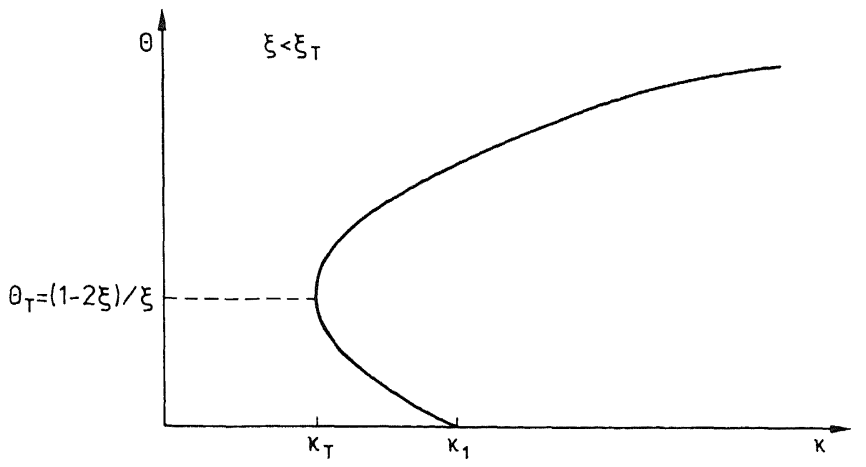

Fig. 1

One easily verifies that this expression defines a decreasing function of $\theta$ which equals $\beta-\mu>0$ for $\theta=0$ and tends to $\beta \xi^{2}-\mu-\alpha$ for $\theta \rightarrow \infty$. So provided

$$
\frac{\beta \xi^{2}}{\mu+\alpha}<1,
$$

there exists precisely one value of $\theta$, say $\theta_{2}$, for which the growth rate equals zero. The corresponding value of $\kappa$ is, by definition, $\kappa_{2}$. Since finding $\theta_{2}$ amounts to solving a quadratic equation, one can derive an explicit expression for $\kappa_{2}$ as given above.

Since $\partial f / \partial \xi>0$ we infer that $\theta_{2}$ is an increasing function of $\xi$. On the other hand, $\theta_{T}=(1-2 \xi) / \xi$ is a decreasing function of $\xi$. By definition $\xi_{p}$ is the value of $\xi$ for which $\theta_{2}=\theta_{T}$. Hence $\xi_{p}$ has to be a solution of

$$
\frac{\alpha+\beta f\left(\frac{1-2 \xi}{\xi}, \xi\right)}{1+\frac{1-2 \xi}{\xi}}-\mu-\alpha=0,
$$

which can be rewritten as a cubic equation in $\xi$.

The condition (3.1) can be interpreted as follows (as was first pointed out by Andreasen [3]): in a population consisting of infectives only, the expected number of offspring of any newborn individual which is infected immediately at birth, is given by $\beta \xi^{2} /(\mu+\alpha)$. Clearly this is a lower bound for the expected number of offspring in any other situation. For a population to be at equilibrium it is necessary that the expected number of offspring equals one. Combining these observations we find that the existence of an equilibrium requires condition (3.1).

\section{Transformation, unification and analysis of the model equations}

In this section we want to introduce a unified formulation for models I and II and analyse the dynamical behaviour of the resulting system. We begin with a transformation of the model equations into different coordinate systems. For the transformation to be useful, it is essential that the birth term $B=B(S, I)$ is homogeneous of degree one, i.e. $B(\alpha S, \alpha I)=\alpha B(S, I)$, and that the force of infection equals the product of the proportion of infectives $I(I+S)$ and a factor depending on total population size $I+S$ only. 
In model I we define

$$
x:=\frac{1}{I+S}, \quad y:=\frac{I}{I+S} .
$$

The transformed system of equations for model $I$ is

$$
\begin{aligned}
& \frac{d x}{d t}=x\left(\mu+\alpha y-\beta(1-(1-\xi) y)^{2}\right), \\
& \frac{d y}{d t}=y\left(\left(\frac{\kappa}{c x+1}-\alpha\right)(1-y)-\beta(1-(1-\xi) y)^{2}\right) .
\end{aligned}
$$

We are interested in the dynamics of this system in the region

$$
M:=\left\{(x, y) \in \mathbb{R}^{2}: x \geqslant 0,0 \leqslant y<b\right\}
$$

with $b=1$.

For model II we take the following transformation

$$
x:=\frac{1}{N}, \quad y:=\frac{P}{N} .
$$

The transformed system of equations for model II is given by

$$
\begin{aligned}
& \frac{d x}{d t}=x\left(\alpha y+\mu-\beta\left(\frac{k}{(1-\xi) y+k}\right)^{k}\right), \\
& \frac{d y}{d t}=y\left(\frac{\kappa}{c x+1}-(\sigma+\alpha)-\frac{\alpha}{k} y-\beta\left(\frac{k}{(1-\xi) y+k}\right)^{k}\right) .
\end{aligned}
$$

For this system we are interested in the dynamics in the whole positive quadrant, so now we define

$$
M:=\left\{(x, y) \in \mathbb{R}^{2}: x \geqslant 0,0 \leqslant y<b\right\}
$$

with $b=\infty$. In (4.2) and (4.4) all parameters are nonnegative and $\beta>\mu$.

We use the following unified notation

$$
\begin{aligned}
& \frac{d x}{d t}=x F(y), \\
& \frac{d y}{d t}=y G(\kappa, x, y),
\end{aligned}
$$

with $(x, y) \in M, \kappa \geqslant 0$. We assume that $F$ and $G$ are continuously differentiable in all variables.

We will proceed as follows: We first state a set of conditions which are satisfied by the special functions $F$ and $G$ defined by the model equations (4.2) and (4.4). We then study for fixed $\kappa$ the steady states of the transformed system and their local stability and we give a catalogue of possible phase portraits of the system. Then we show how with changing $\kappa$ the phase portrait changes with the bifurcation of steady states and periodic solutions. In Subsects. 4.1-4.3 the parameter $\xi$ will be fixed and suppressed in the notation, while in Subsect. 4.4 we will investigate how the dynamics depends on $\xi$. Finally in Subsect. 4.5 we demonstrate how exponential solutions of the original model equations are related to steady states of the transformed system. 


\subsection{Conditions on $F$ and $G$}

We assume that $F$ and $G$ satisfy the following conditions:

$$
\text { I }\left\{\begin{array}{l}
\text { (i) } \frac{d F}{d y}>0 ; \quad \exists ! \bar{y} \in[0, b): F(\bar{y})=0 \\
\text { (ii) } \frac{\partial G}{\partial x}<0 \text { and there exists a unique function } g=g(\kappa, y) \\
\text { such that } G(\kappa, g(\kappa, y), y)=0 \\
\text { (iii) } \frac{\partial G}{\partial \kappa}>0, \quad G(0, x, y)<0 \quad \forall(x, y) \in M .
\end{array}\right.
$$

We will alternatively use the notation $F^{\prime}$ for the derivative of $F$ and $G_{\kappa}, G_{x}, G_{y}$ for the partial derivatives of $G$.

It is easy to see that the transformed systems (4.2) and (4.4) fulfil conditions I(ii), (iii) ( see Appendix). For model II condition I(i) is always fulfilled, provided $\beta>\mu$. For model I the condition $\bar{y} \in[0, b)$ leads to the condition

$$
\frac{\beta \xi^{2}}{\mu+\alpha}<1
$$

as a necessary condition for the existence of a steady state.

The isoclines $d x / d t=0$ are the $y$-axis and a straight line given by $y=\bar{y}$. The isoclines $d y / d t=0$ are the $x$-axis and the graph of $g$, where $g$ is the solution of the implicit equation $G(\kappa, x, y)=0$. Condition I(ii) shows that $G(\kappa, x, y)<0$ for $x>g(\kappa, y)$, and $G(\kappa, x, y)>0$ for $x<g(\kappa, y)$. The graph of $g$ does not necessarily intersect the region $M$, in fact condition I(iii) ensures that it does not for $\kappa=0$. But the restriction of our attention to $M$ was only motivated by the biological interpretation, while mathematically we can study system (4.5) in $\mathbb{R}^{2}$. It is easy to see that condition I(ii) holds in $\mathbb{R}^{2}$ for the functions considered in (4.2) and (4.4).

We may get three types of steady states:

1. The trivial steady state $x=0, y=0$.

2. Nontrivial steady states $\left(0, y^{*}\right)$ on the $y$-axis determined by $g(\kappa, y)=0$.

3. A unique positive steady state given by $y=\bar{y}$ and $\bar{x}:=g(k, \bar{y})$.

The following set of conditions yields constraints on the form of the function $g$ and on how this form depends on the parameter $\kappa$.

$$
\text { II }\left\{\begin{array}{l}
\text { (i) } g_{y} \text { is independent of } \kappa \text {. } \\
\text { (ii) } g_{y}(\kappa, y) \text { as a function of } y \text { has at most one zero. When } \\
\text { the zero exists, it corresponds to a local maximum of } g . \\
\text { (iii) } \lim _{y \rightarrow b} g(\kappa, y)<0 .
\end{array}\right.
$$

Note that these assumptions imply that $g$ can have no local minimum.

\subsection{A catalogue of phase portraits}

In this section we want to study which phase portraits are possible for system (4.5) under the conditions stated in Subsect. 4.1. We consider $\kappa$ as fixed and 
consequently suppress it in the notation of this section. We first show that positive orbits are always bounded.

Proposition 1 The set $M$ is positively invariant and there exists a bounded region $Q$ which absorbs all orbits. When $g$ has no zero to the right of $\bar{y}$ all orbits converge to the segment of the $y$-axis between 0 and $\bar{y}$. When $g$ has no zero at all, $(0,0)$ is globally asymptotically stable.

Proof. The invariance of $M$ is a direct consequence of assumption II(iii). Now choose a $\tilde{y}$ with $\bar{y}<\tilde{y}<b$, such that $g(y)<0$ for $y \geqslant \tilde{y}$. So along the straight line $y=\tilde{y}$ we have $G(x, \tilde{y})<0$. Choose

Define $c>0$ by

$$
\tilde{x}>\sup _{0 \leqslant y<b} g(y)
$$

$$
-c=\max \{G(\tilde{x}, y): \bar{y} \leqslant y \leqslant \tilde{y}\} .
$$

Let $S$ denote the semi-infinite strip

$$
S=\{(x, y): x \geqslant \tilde{x}, \bar{y} \leqslant y \leqslant \tilde{y}\} .
$$

Assumption I(ii) implies that $G(x, y) \leqslant-c$ for $(x, y) \in S$. Denote by $t \mapsto \gamma(t)$ the trajectory with $\gamma(0)=(\tilde{x}, \tilde{y})$. Since $F(\tilde{y})>0$ and $G(\tilde{x}, \tilde{y})<0$ the trajectory $\gamma$ enters the strip $S$. As long as it stays in $S$ we have

$$
\frac{d y}{d t} \leqslant-c y \text { and } \frac{d x}{d t}>0 .
$$

So in finite time $\gamma$ hits the boundary $y=\bar{y}$ of $S$, say at $x=\bar{x}$.

Consider the region $Q$ bounded by the $x$ - and $y$-axis, the straight lines $x=\bar{x}$ and $y=\tilde{y}$, and the part of $\gamma$ inside $S$. Note that $\gamma$ stays in $Q$ after hitting $(\bar{x}, \bar{y})$, since $d x / d t<0$ for $y<\bar{y}$. So the boundary segments of $Q$ are either invariant or such that the vector field points inward along them. We conclude that $Q$ is positively invariant (see Fig. 2).

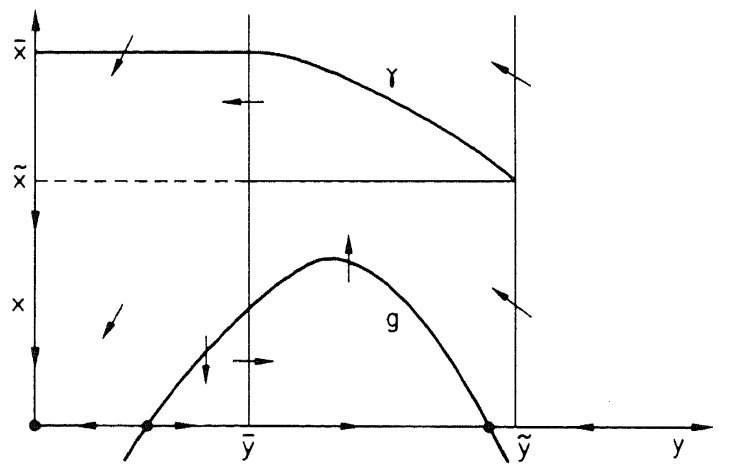

Fig. 2

Next consider any trajectory starting in $S$ but not in $Q$. By the same argument as before it hits the line $y=\bar{y}$ in finite time, somewhere above $\bar{x}$. It then enters the region $\{(x, y): x>\bar{x}, 0<y<\bar{y}\}$, where $d x / d t<0$ and $d y / d t<0$. Inevitably it then enters in the long run $Q$ at the boundary $x=\bar{x}$.

Finally, consider any trajectory starting with $y>\tilde{y}$. Using now $c$ defined by

$$
-c=\sup \{G(0, y): \bar{y} \leqslant y<b\}
$$


and the same argument as before we conclude that the trajectory has to hit the line $y=\tilde{y}$ in finite time. So it either enters $Q$, and we are ready, or it enters $S$ and we can invoke the result above that trajectories starting in $S$ have to enter $Q$.

When $g(y)<0$ for all $y>\bar{y}$ we find, using exactly the same arguments, that the strip $\{(x, y): x \geqslant 0,0 \leqslant y<\bar{y}\}$ is positively invariant and absorbing. In the strip we have $F(y)<0$ and consequently $x(t) \rightarrow 0$ for $t \rightarrow \infty$. When $g$ has no zero at all, then $d y / d t<0$ and consequently $y(t) \rightarrow 0$ as well.

This completes the proof.

We now analyse the local stability of the different types of steady states. The Jacobian of system (4.5) is given by

$$
J_{(x, y)}=\left(\begin{array}{cc}
F(y) & x F^{\prime}(y) \\
y G_{x}(x, y) & y G_{y}(x, y)+G(x, y)
\end{array}\right) .
$$

In the trivial steady state we get

$$
J_{(0,0)}=\left(\begin{array}{cc}
F(0) & 0 \\
0 & G(0,0)
\end{array}\right) .
$$

From condition I(i) it follows that $F(0)<0$, so the stability is determined by the sign of $G(0,0)$ and hence by the sign of $g(0)$. In case $g(0)<0$ the trivial steady state is a stable node and in case $g(0)>0$ it is a saddle point.

The Jacobian for an equilibrium $\left(0, y^{*}\right)$, i.e. an equilibrium on the positive $y$-axis, is given by

$$
J_{\left(0, y^{*}\right)}=\left(\begin{array}{cc}
F\left(y^{*}\right) & 0 \\
y^{*} G_{x}\left(0, y^{*}\right) & y^{*} G_{y}\left(0, y^{*}\right)
\end{array}\right)
$$

and consequently the eigenvalues are $F\left(y^{*}\right)$ and $y^{*} G_{y}\left(0, y^{*}\right)$. The sign of the first eigenvalue is determined by the position of $y^{*}$ relative to the zero $\bar{y}$ of $F$. More precisely we have $F\left(y^{*}\right)<0$ for $y^{*}<\bar{y}$, and $F\left(y^{*}\right)>0$ for $y^{*}>\bar{y}$. From the implicit function theorem it follows that the sign of $G_{y}$ (and therefore the sign of the second eigenvalue) is the same as the sign of $g^{\prime}$. So we have the following cases:

$$
\left(0, y^{*}\right) \text { is }\left\{\begin{array}{l}
\text { a saddle point for } y^{*}<\bar{y} \text { and } g^{\prime}\left(y^{*}\right)>0, \\
\text { a stable node for } y^{*}<\bar{y} \text { and } g^{\prime}\left(y^{*}\right)<0, \\
\text { a saddle point for } y^{*}>\bar{y} \text { and } g^{\prime}(y)<0, \\
\text { an unstable node for } y^{*}>\bar{y} \text { and } g^{\prime}\left(y^{*}\right)>0 .
\end{array}\right.
$$

The Jacobian at the positive equilibrium $(\bar{x}, \bar{y})$ is given by

$$
J_{(\bar{x}, \bar{y})}=\left(\begin{array}{cc}
0 & \bar{x} F^{\prime}(\bar{y}) \\
\bar{y} G_{x}(\bar{x}, \bar{y}) & \bar{y} G_{y}(\bar{x}, \bar{y})
\end{array}\right) .
$$

The assumptions I(i), (ii) imply that the determinant is positive and consequently the stability of the positive steady state depends on the sign of the trace $G_{y}(\bar{x}, \bar{y})$ which is again identical to the sign of $g^{\prime}(\bar{y})$. If $g^{\prime}(\bar{y})<0$ we have a sink, if $g^{\prime}(\bar{y})>0$ a source. In both cases we can have a node or a spiral point.

In the following we discuss the various possible phase portraits of system (4.5). In the simple case that $g(y)<0$ for all $y \geqslant 0$ the trivial steady state is the only equilibrium and all solutions converge to it for $t \rightarrow \infty$ (Proposition 1). Now assume that $g$ takes positive values on $[0, b)$.

The sign of $g(0)$ then determines whether we have one or two nontrivial steady states on the $y$-axis and whether or not $(0,0)$ is stable. The sign of $g(\bar{y})$ 
determines whether or not we have an interior steady state and, in case it exists, its stability is determined by the sign of $g^{\prime}(\bar{y})$. Likewise the sign of $g(\bar{y})$ determines the position of the nontrivial steady states on the $y$-axis relative to $\bar{y}$, which in turn has a decisive influence on their stability. Excluding degenerate cases Figs. 3-9 list the collection of possible phase portraits classified by the binary three vector $\left(\operatorname{sign} g(0)\right.$, sign $g(\bar{y})$, sign $\left.g^{\prime}(\bar{y})\right)$.

Note that the case $(+,-,+)$ cannot occur under assumptions II(ii), (iii). To conclude this section we make an observation concerning a case which is ruled out by assumption I(i), but which is, nevertheless, relevant in the context
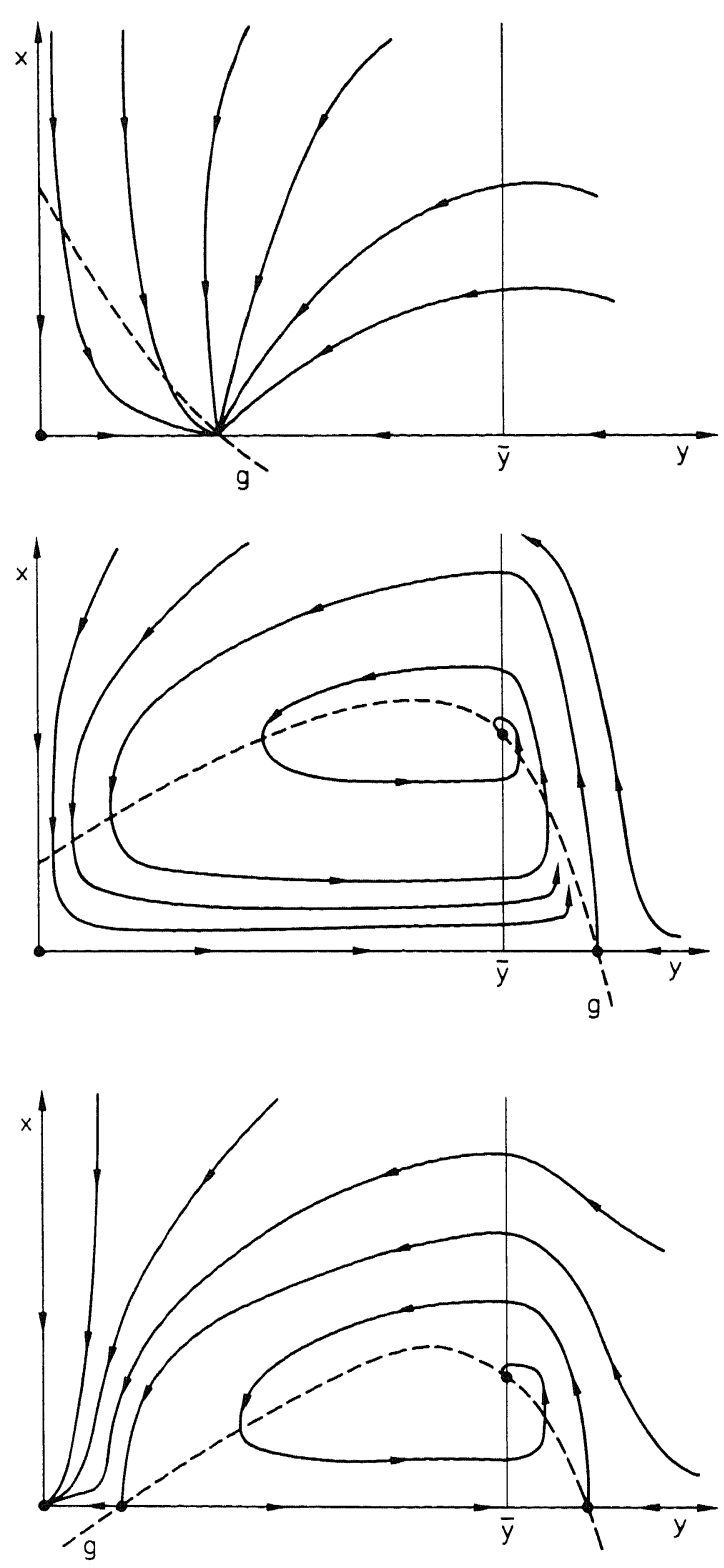

Fig. 3. $(+,-,-)$ The unique nontrivial steady state on the $y$-axis is globally stable in $M-\{(x, y): y=0\}$
Fig. 4. $(+,+,-)$ The interior steady state is locally stable, but in principle there can be an even number of limit cycles surrounding it

Fig. 5. $(-,+,-)$ The interior steady state is stable, but there can be an even number of limit cycles surrounding it. The stable manifold of the lower one of the nontrivial steady states on the $y$-axis separates the domains of attraction of the $(0,0)$ stable steady state and (the outer limit cycle surrounding) the interior steady state. We have bistable behaviour 
(a)

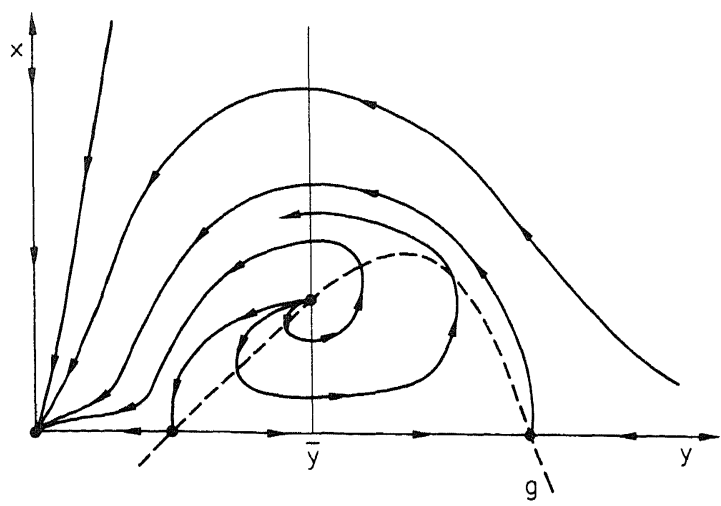

(b)

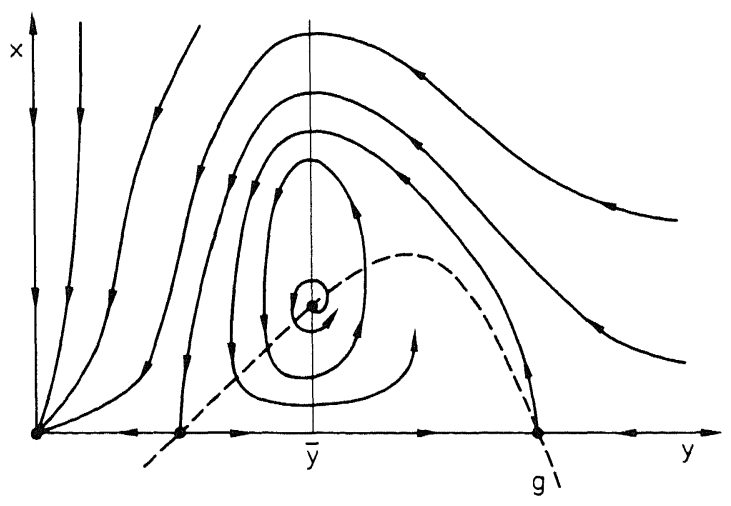

Fig. 6. (a) $(-,+,+)$-eradication, (b) $(-,+,+)$-bistability. The $(-,+,+)$ case splits into two subcases, depending on the behaviour of the stable manifold of the lower one of the two nontrivial steady states on the $y$-axis. When the stable manifold "originates" at the $(y=b)$-boundary of $M$, it acts as a separatrix and consequently the unstable interior steady state must be surrounded by an odd (so at least one!) number of limit cycles. When the stable manifold is contained in a compact subset of $M$, it either extends back to the unstable interior point or to the outer one of an even number of limit cycles surrounding it. In the first situation every other orbit starting in the interior must converge to the trivial steady state and in the second situation the same is true except for the orbits inside the outer limit cycle. The two subcases are separated from each other by the degenerate case in which the unstable manifold of the upper steady state on the $y$-axis coincides with the stable manifold of the lower one (i.e. there exists a heteroclinic orbit or saddle-connection)

Fig. 7. $(+,+,+)$ The unstable manifold of the upper saddle point on the $y$-axis cannot connect to the trivial saddle point or to itself, nor can it converge to the unstable interior steady state. Consequently its $\omega$-limit set must contain a limit cycle. We conclude that an odd number of limit cycles must surround the interior steady state

of model I when assumption (3.1) does no hold. When $F(y)<0$ for all $y \in[0, b)$ necessarily $x(t) \rightarrow 0$ as $t \rightarrow \infty$ and we can concentrate on the $y$-axis when investigating the asymptotic dynamics. The cases $(+,-,-)$ and $(-,-,-)$ describe the two possibilities adequately. 

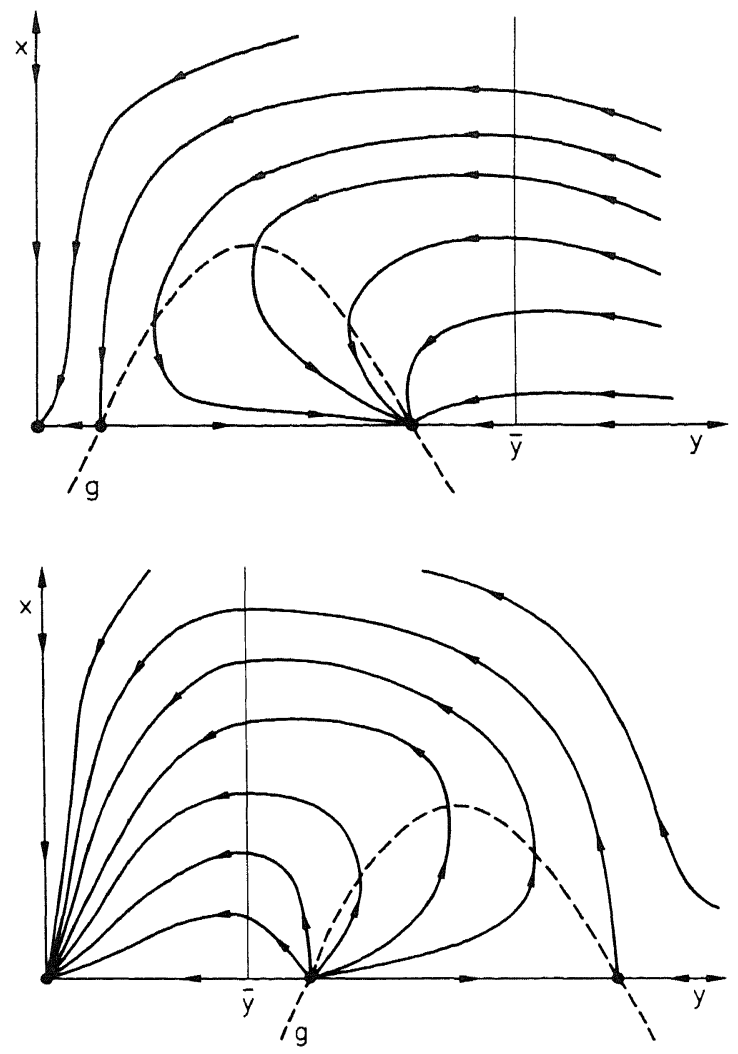

Fig. 8. $(-,-,-)$ This is a bistable case with the stable manifold of the lower nontrivial steady state on the $y$-axis separating the domains of attraction of the trivial steady state and the stable upper steady state on the $y$-axis

\subsection{Bifurcation diagrams}

Fig. 9. $(-,-,+)$ All orbits starting in the interior of $M$ converge to the trivial steady state

We now take into account the contact rate parameter $\kappa$ and study how the phase portrait changes with increasing $\kappa$. From assumptions I(ii) and I(iii) we conclude that $\partial g / \partial \kappa>0$, which means that the graph of $g$ moves in the positive $x$-direction as $\kappa$ increases. In our notation this means that, as $\kappa$ increases, the first and second component of the sign-vector can change from - to + , but not vice versa. Assumption II(i) guarantees that sign $g_{y}(\kappa, \bar{y})$ does not depend on $\kappa$ or, in other words, that the stability character of the interior steady state does not change when $\kappa$ is varied. In our notation it implies that the third component of the sign-vector stays constant as $\kappa$ varies. We start from the situation in which $g<0$ for all $y \geqslant 0$ (so that $(0,0)$ is globally stable). We shall denote this situation symbolically by $(-)$. Depending on the exact form of the graph of $g$ we obtain one of the following sequences of transitions as $\kappa$ is increased. (Recall that our three vector notation implicitly includes the assumption that $g$ assumes positive values on $[0, b)$.)

Diagram $1(-) \rightarrow(+,-,-) \rightarrow(+,+,-)$

$g(\kappa, y)$ as a function of $y$ is strictly monotone decreasing. With increasing $\kappa$ we then first for a threshold value $\kappa_{1}$ have $g\left(\kappa_{1}, 0\right)=0$ and a stable node bifurcates from the trivial solution. For a threshold value $\kappa_{2}$, characterized by $g\left(\kappa_{2}, \bar{y}\right)=0$, we get a bifurcation of a positive stable steady state (see Fig. 10). 


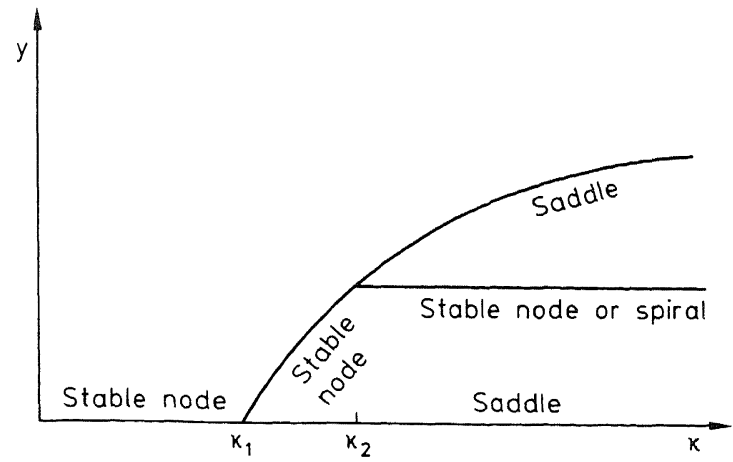

Fig. 10. Bifurcation diagram 1 in the $\kappa-y$-plane

Diagram 2 (a) $(-) \rightarrow(-,-,-) \rightarrow(+,-,-) \rightarrow(+,+,-)$

(b) $(-) \rightarrow(-,-,-) \rightarrow(-,+,-) \rightarrow(+,+,-)$

$y \mapsto g(\kappa, y)$ has a maximum at $y_{\max }<\bar{y}$. Then we first have a bifurcation of two steady states, a saddle and a stable node, at $\kappa=\kappa_{T}$ defined by $g\left(\kappa_{T}, y_{\max }\right)=0$. At $\kappa=\kappa_{1}$ there is a subcritical bifurcation as the saddle passes the trivial equilibrium. At $\kappa=\kappa_{2}$ a stable node or spiral point bifurcates from the existing branch of stable nodes (see Figs. 11(a), (b)). If $\kappa_{1}<\kappa_{2}$, we have case (a), otherwise (b).
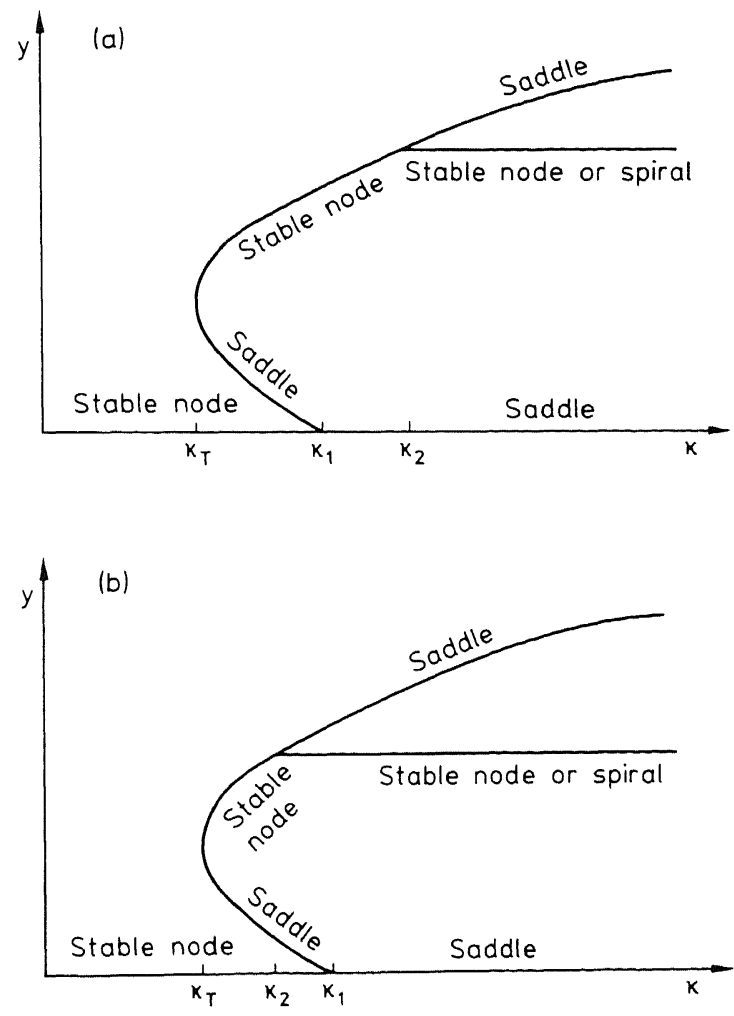

Fig. 11. Bifurcation diagram 2 for: (a) $\kappa_{1}<\kappa_{2}$, (b) $\kappa_{1}>\kappa_{2}$ 
Diagram $3(-) \rightarrow(-,-,+) \rightarrow(-,+,+)_{\text {eradication }}$

$$
\rightarrow(-,+,+)_{\text {bistable }} \rightarrow(+,+,+)
$$

$y \mapsto g(\kappa, y)$ has a maximum $y_{\max } \in M$ with $y_{\max }>\bar{y}$. Again as $\kappa=\kappa_{T}$ two steady states bifurcate, a source $y_{1}$ and a saddle $y_{2}$. The unstable manifold of the saddle $y_{2}$ connects to the trivial steady state, to which all positive solutions converge (see Fig. 9). At $\kappa=\kappa_{2}$ an unstable positive equilibrium bifurcates from $y_{1}$, which turns into a saddle. Still all positive solutions converge to $(0,0)$ except for a heteroclinic orbit connecting the positive steady state with the saddle $y_{1}$ (see Fig. $6(\mathrm{a}))$. At $\kappa=\kappa_{p}$ the unstable manifold of $y_{2}$ connects to $y_{1}$ and we have a bifurcation of a limit cycle (see Fig. 6(b)). The existence of the limit cycle will be proved in Proposition 2. At $\kappa=\kappa_{1}$ we have a subcritical bifurcation as $y_{1}$ crosses the trivial steady state. We have $\kappa_{2}<\kappa_{p}<\kappa_{1}$. Figure 12 shows the bifurcation diagram for this situation.

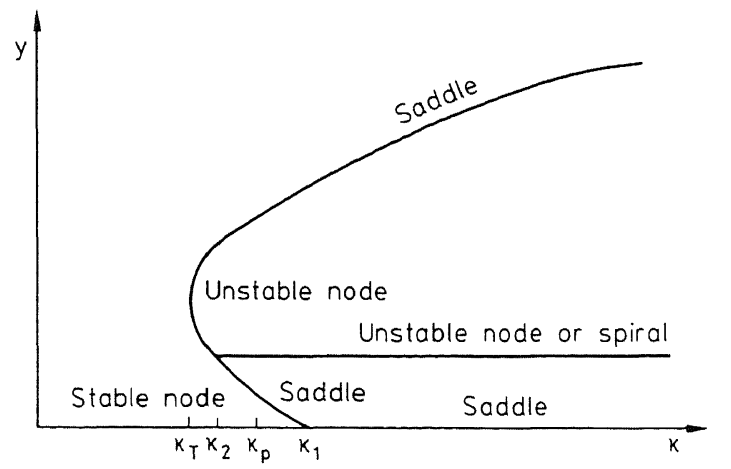

Fig. 12. Bifurcation diagram 3

That in Diagram 3 in going from $(-,-,+)$ to $(-,+,+)$ we first obtain the eradication situation follows from a continuity argument: at the bifurcation the unstable manifold of the upper steady state on the $y$-axis still connects to the trivial steady state. Likewise, in going from $(-,+,+)$-eradication to $(+,+,+)$ we must go through $(-,+,+)$-bistable in order to obtain the odd number of limit cycles. In principle, when $\kappa$ increases one may go a number of times back and forth between $(-,+,+)$-eradication and $(-,+,+)$-bistable.

Proposition 2 Assume the situation of Diagram 3: $g(\kappa, y)$ has a maximum at $y_{\max }>\bar{y}$ and two zeros $y_{1}$ and $y_{2}$ on the positive $y$-axis. Then there exists a $\kappa_{p}$ with $\kappa_{2}<\kappa_{p} \leqslant \kappa_{1}$ such that for all $\kappa>\kappa_{p}$ there exists a limit cycle in $M$. The limit cycle comes into existence by a global bifurcation from a saddle connection.

Proof. For $\kappa \searrow \kappa_{1}$ we have $y_{1} \searrow 0$. From Proposition 1 it follows that the unstable manifold of the saddle $\left(0, y_{2}\right)$ in $M$ is bounded. If we denote the unstable manifold of $\left(0, y_{2}\right)$ by $\gamma$, the $\omega$-limit set of $\gamma$ lies in the region $Q$ defined in the proof of Proposition 1. Since the interior steady state is unstable, the $\omega$-limit set of $\gamma$ must be an equilibrium on the $y$-axis or a limit cycle.

For $\kappa=\kappa_{1}$ the trivial steady state $(0,0)$ is the only other equilibrium on the $y$-axis. So if there is no limit cycle, $(0,0)$ must be the $\omega$-limit set of $\gamma$. As soon as $\kappa>\kappa_{1}$ the trivial equilibrium turns into a saddle with the $x$-axis as a stable manifold. Consequently $(0,0)$ can no longer be the $\omega$-limit set of $\gamma$ and there must exist a limit cycle in $Q$. If we denote the value of $\kappa$ for which the limit cycle comes into existence by $\kappa_{p}$, we conclude that $\kappa_{p} \leqslant \kappa_{1}$. 
On the other hand for $\kappa=\kappa_{2}$ the unstable steady state bifurcates in a nondegenerate bifurcation, so $\kappa_{p}$ must be larger than $\kappa_{2}$. The positive steady state is always unstable. This means that the limit cycle does not arise through Hopf-bifurcation, but comes into existence through a global bifurcation from the saddle connection of $\left(0, y_{1}\right)$ and $\left(0, y_{2}\right)$.

We recall from Subsect. 4.2 that the threshold $\kappa_{1}$ is characterized by

$$
G\left(\kappa_{1}, 0,0\right)=0
$$

or, equivalently, $g\left(\kappa_{1}, 0\right)=0$, and $\kappa_{2}$ is characterized by $g\left(\kappa_{2}, \bar{y}\right)=0$, while $\kappa_{T}$ is characterized by $g\left(\kappa_{T}, y_{\max }\right)=0$. The threshold value $\kappa_{p}$ is only implicitly defined in terms of the existence of a saddle connection (in fact several such values may exist, each one corresponding to the birth or death of a limit cycle). The threshold value $\kappa_{0}$, which distinguishes in the original model equations between an eventual extinction of the disease and the dilution effect situation, is characterized by $F(0)=G\left(\kappa_{0}, 0,0\right)$. This can be seen in the following way. The linearized system in the trivial steady state is given by

$$
\left(\begin{array}{l}
\dot{x} \\
\dot{y}
\end{array}\right)=J_{(0,0)}\left(\begin{array}{l}
x \\
y
\end{array}\right) .
$$

The solutions for an initial value $\left(x_{0}, y_{0}\right)$ are given by

$$
x(t)=x_{0} e^{\lambda_{1} t} \text { and } y(t)=y_{0} e^{\lambda_{2} t}
$$

with

$$
\lambda_{1}=F(0) \quad \text { and } \quad \lambda_{2}=G(\kappa, 0,0) .
$$

For $\kappa<\kappa_{0}$ we have $\lambda_{2}<\lambda_{1}<0$. This implies

$$
\frac{y}{x}=\frac{y_{0}}{x_{0}} e^{\left(\lambda_{2}-\lambda_{1}\right) t} \rightarrow 0 \text { for } t \rightarrow \infty
$$

For $\kappa_{0}<\kappa<\kappa_{1}$ we have $\lambda_{1}<\lambda_{2}<0$, which implies

$$
\frac{y}{x}=\frac{y_{0}}{x_{0}} e^{\left(\lambda_{2}-\lambda_{1}\right)^{t}} \rightarrow \infty \text { for } t \rightarrow \infty .
$$

Since both $I$ and $P$ are given by $y / x$, we conclude that the disease dies out when $\kappa<\kappa_{0}$, whereas when $\kappa>\kappa_{0}$ the number of infectives/parasites grows exponentially.

We have now analysed the dynamics of the system (4.5) in dependence of the parameter $\kappa$, while taking all other parameters in the models to be fixed. In the following subsection we depart from this assumption and analyse how the dynamics of the system change with $\xi$ varying in the interval $[0,1]$. In doing so we concentrate more on the specific systems (4.2) and (4.4) corresponding to models I and II than on the general system (4.5).

\subsection{Dependence on the parameter $\xi$}

In Subsect. 4.3 we distinguished three bifurcation diagrams. Which of these is realized depends on the value of the parameter $\xi$ since $\xi$ determines the exact form of $g(\kappa, y)$. With decreasing $\xi$ we move from diagram 1 to 2 to 3 .

First we show that there exists a threshold value $\xi=\xi_{T}$ such that the bifurcation of steady states from the trivial steady state with $\kappa$ as a bifurcation parameter 
is subcritical for $\xi<\xi_{T}$ and supercritical for $\xi>\xi_{T}$. The branch of steady states on the $y$-axis is described by the implicit function

$$
G(\kappa, 0, y)=0,
$$

which we solve for $\kappa$ to get a function $\kappa=\kappa(y)$. This is possible because of condition I(iii). The direction of bifurcation is then determined by the sign of the derivative $d \kappa / d y$ evaluated at $y=0$.

For model I we get

$$
\kappa(y)=\frac{\beta(1-(1-\xi) y)^{2}}{1-y}+\alpha .
$$

This implies

$$
\left.\frac{d \kappa}{d y}\right|_{y=0}=\beta(2 \xi-1)
$$

and we infer that the threshold for model I is $\xi_{T}=\frac{1}{2}$.

For model II we get

$$
\kappa(y)=\sigma+\alpha+\frac{\alpha}{k} y+\beta\left(\frac{k}{(1-\xi) y+k}\right)^{k}
$$

and

$$
\left.\frac{d \kappa}{d y}\right|_{y=0}=\frac{\alpha}{k}-\beta(1-\xi) .
$$

So for model II we get the threshold $\xi_{T}=1-(\alpha / \beta k)$.

Proposition 3 For both models limit cycles do not exist for $\xi>\xi_{T}$. Local stability of a steady state then implies global stability (with respect to $M^{0}$, the interior of $M)$.

Proof. To show the nonexistence of limit cycles we use a theorem by Dulac (see [19]). We first consider model I and define*

$$
B(x, y):=\frac{1}{x y(1-y)} .
$$

Then easy computation shows that

$$
\begin{array}{r}
\frac{\partial}{\partial x}(B(x, y) x F(y))+\frac{\partial}{\partial y}(B(x, y) y G(\kappa, x, y)) \\
=-\frac{\beta(1-(1-\xi) y)}{x(1-y)^{2}}((1-\xi) y+2 \xi-1)
\end{array}
$$

which is negative for $\xi>\xi_{T}=\frac{1}{2}$ for all $(x, y) \in M^{0}$.

In case of model II we define

$$
B(x, y):=\frac{1}{x y} .
$$

\footnotetext{
* We thank Dr. Jin Cheng-Fu for suggesting this auxiliary function
} 
Then we have

for all $(x, y) \in M$. For model II we have

$$
\frac{\partial}{\partial x}(B(x, y) x F(y))+\frac{\partial}{\partial y}(B(x, y) y G(\kappa, x, y))=\frac{1}{x} G_{y}(\kappa, x, y)
$$

$$
G_{y}(\kappa, x, y)=-\frac{\alpha}{k}+\beta\left(\frac{k}{(1-\xi) y+k}\right)^{k} \frac{k(1-\xi)}{(1-\xi) y+k} .
$$

We see that $G_{y}<0$ is equivalent to

$$
\frac{\alpha}{k}>\beta\left(\frac{k}{(1-\xi) y+k}\right)^{k} \frac{k(1-\xi)}{(1-\xi) y+k} .
$$

The right hand side of this inequality is strictly decreasing in $y$. This means that if the inequality is fulfilled for $y=0$, it is valid for all $y \geqslant 0$. For $y=0$ the inequality reduces to

$$
\frac{\alpha}{k}>\beta(1-\xi)
$$

which is fulfilled if and only if $\xi>\xi_{T}$. So for $\xi>\xi_{T}$ we have $G_{y}<0$ for all $(x, y) \in M^{0}$.

We can now conclude for both models by using a theorem of Dulac that the system (4.5) does not have any closed trajectory or singular closed trajectory lying entirely in $M$.

For $\xi>\xi_{T}$ we have a supercritical bifurcation of a steady state from the trivial steady state. This means that we have the situation described in Subsect. 4.3, diagram 1. For $\kappa<\kappa_{1}$ the trivial steady state is the only equilibrium and it is locally stable. Boundedness of solutions implies global stability. For $\kappa>\kappa_{1}$ there exists one locally stable equilibrium; the other existing equilibria are saddles, whose stable manifolds conicide with parts of the boundary of $M$. This means that all solutions starting in the interior of $M$ converge to the locally stable equilibrium (here we use once more their boundedness).

Before we prove the following proposition, recall that for $\xi<\xi_{T}$ we denoted by $\kappa_{T}$ the threshold value for $\kappa$ for which two steady states $y_{1}$ and $y_{2}$ arose from a saddle node bifurcation on the $y$-axis. In the previous subsections we always assumed that $\xi$ was fixed. Now we have to take into account that $\kappa_{r}$ varies with $\xi$. We assume the following set of conditions to hold:

$$
\text { III }\left\{\begin{array}{l}
\text { (i) } \frac{\partial F}{\partial \xi}<0 \\
\text { (ii) } \frac{d}{d \xi} y_{\max }<0 \text { for } \xi<\xi_{T} \\
\text { (iii) } \xi=0 \Rightarrow \bar{y}<y_{\max } .
\end{array}\right.
$$

Condition III(iii) ensures that for $\xi=0$ the situation described in Subsect. 4.3, diagram 3 is realized. For model I condition III(iii) is fulfilled, because $g_{y}(\kappa, y)>0$ for $\xi=0$, so we have $y_{\max }=1$. For model II condition III(iii) is fulfilled, provided $\alpha / \kappa \beta$ is small enough. More precisely, there exists a constant $\varepsilon<1$ such that condition III(iii) is fulfilled if and only if $(\alpha / k \beta)<\varepsilon$ (see Appendix). Biologically this means that the mortality caused by the parasites should be small compared with the birth rate of the host population weighted by the amount of "clumping" of the parasites. 
Proposition 4 There exists a threshold value $\xi_{p}$ such that for decreasing $\xi$ there occurs a Hopf-bifurcation of a limit cycle from the positive stable equilibrium as $\xi$ crosses $\xi_{p}$, for any fixed $\kappa>\kappa_{T}$ with $\kappa_{T}=\kappa_{T}\left(\xi_{p}\right)$.

Proof. From I(i) and III(i) it follows that $(d / d \xi) \bar{y}>0$, i.e. if $\xi$ decreases then also $\bar{y}$ decreases. On the other hand III(ii) shows that with decreasing $\xi$ the maximum of the function $g$ moves in the positive $y$-direction, i.e. $y_{\max }$ increases, starting from $y_{\max }=0$ for $\xi=\xi_{T}$. The interior steady state loses its stability and the periodic solution comes into existence at the moment that $\bar{y}=y_{\max }$. Condition III(iii) ensures that there exists a $\xi_{p} \in\left[0, \xi_{T}\right.$ ) for which $\bar{y}=y_{\max }$. Conditions III(i), (ii) guarantee that $\bar{y}$ crosses $y_{\max }$ with positive speed as $\xi$ decreases. This proves that there occurs a Hopf-bifurcation at $\xi=\xi_{p}$.

\subsection{Relation between original and transformed systems}

We now want to close the circle and show how the results for the transformed system can be interpreted in terms of the original variables. To this end we demonstrate how exponentially growing solutions of the original equations (2.3) and (2.10) are related to steady states of the transformed system (4.5). With the term "exponential solution" we refer to a solution for which both (sub)populations in the original equations grow eventually exponentially with the same growth rate $\lambda$. Solutions with this type of asymptotic behaviour can exist for both models, since for growing populations the infection rate $\varphi$ converges to a function that is homogeneous of degree 0 , i.e. a function that is invariant under scaling of $S$ and $I$ (or $N$ and $P$, respectively) with the same factor. Indeed, for $c=0$ the function $\varphi$ is homogeneous of degree 0 . For $c>0$ we call it asymptotically homogeneous.

If in Eqs. (2.3) and (2.10) we set $c=0$, there exist solutions of the form

$$
S(t)=S^{*} e^{i t}, \quad I(t)=I^{*} e^{i t}
$$

for (2.3), and solutions of the form

$$
N(t)=N^{*} e^{\lambda t}, \quad P(t)=P^{*} e^{\lambda t}
$$

for (2.10) with some $\lambda \in \mathbb{R}$ for $\kappa$ larger than some threshold $\kappa_{T}$. For these exponential solutions $\varphi$ is constant in time.

It is clear that for solutions of (2.3) with $I+S \rightarrow \infty$ and solutions of (2.10) with $N \rightarrow \infty$ the infection rate $\varphi$ converges to a function which is homogeneous of degree 0. Furthermore exponential solutions of the homogeneous system (characterized by $c=0$ ) are mapped by the transformations (4.1) and (4.3), respectively, on solutions of $(4.5)$ (with $c=0$ ), which approach a steady state with $x=0$ as $t \rightarrow \infty$.

Assume now that $\left(0, y^{*}\right)$ with $y^{*}>0$ is an asymptotically stable steady state of (4.5). The linearized system in $\left(0, y^{*}\right)$ is given by

$$
\left(\begin{array}{c}
\dot{x} \\
\dot{y}
\end{array}\right)=\left(\begin{array}{cc}
F\left(y^{*}\right) & 0 \\
y^{*} G_{x}\left(\kappa, 0, y^{*}\right) & y^{*} G_{y}\left(\kappa, 0, y^{*}\right)
\end{array}\right)\left(\begin{array}{c}
x \\
y-y^{*}
\end{array}\right) .
$$


We assume that $\lambda_{1}:=F\left(y^{*}\right)<0$ and $\lambda_{2}:=y^{*} G_{y}\left(\kappa, 0, y^{*}\right)<0$. We write $a:=y^{*} G_{x}\left(\kappa, 0, y^{*}\right)$. Solving for an initial value $\left(x_{0}, y_{0}\right)$ we get

$$
\begin{gathered}
x(t)=x_{0} e^{\lambda_{1} t} \\
y(t)=\frac{a}{\lambda_{1}-\lambda_{2}} e^{\lambda_{1} t}+\left(y_{0}-\frac{a x_{0}}{\lambda_{1}-\lambda_{2}}-y^{*}\right) e^{\lambda_{2} t}+y^{*} .
\end{gathered}
$$

This implies that $y(t) \rightarrow y^{*}$ for $t \rightarrow \infty$ and therefore

$$
\frac{y}{x} \sim y^{*} e^{-\dot{\lambda}_{1} t}
$$

This means that asymptotically $y / x$ grows exponentially with the same rate $-\lambda_{1}$ as does $1 / x$.

Furthermore it is easy to see that $x(t)=x_{0} e^{\lambda_{1} t}, y(t)=y^{*}$ is an exact solution of the system (4.5) with $c=0$. This shows that in a neighbourhood of a steady state solutions of the homogeneous system approximate solutions of the inhomogeneous system with an error that depends on the chosen neighbourhood.

This in turn shows that exponential solutions with positive growth rate of the systems (2.3) and (2.10) with $c=0$ approximate solutions of the nonhomogeneous system $(c>0)$ for $t \rightarrow \infty$.

When $\left(0, y^{*}\right)$ is unstable (either a saddle point or a source), the linearization yields an approximation which loses its significance for $t$ tending to infinity. One can then describe the "repelling" behaviour of the steady state with infinite total/host population size and fraction of infectives/mean parasite load $y^{*}$ in terms of exponential solutions, but this is hardly of any interest. The fact that for $c=0$ we again find an exact exponential solution of the nonlinear problem does not alter this conclusion.

The growth rate $-F\left(y^{*}\right)$ of the exponential solutions is the reduced growth rate that was mentioned in Subsect. 3.1, case 3 and in Subsect. 3.2, cases 3 and 4. Furthermore we can see the following: For exponential solutions in the case $c=0$ we have $\varphi=\kappa y^{*}$. This means that increasing $\varphi$ implies increasing $y^{*}$. From condition I(i) we now get that the growth rate of the population decreases as $\varphi$ increases. If we consider $\varphi$ as a parameter, we get a branch of exponential solutions in the $\kappa-\varphi$ plane. Along this branch the growth rate $\lambda$ is decreasing. At the point where $\lambda=0$ the branch of exponential solutions of the homogeneous system intersects a branch of equilibria for the system with $c>0$. For the homogeneous system $\lambda=0$ occurs only at a single point of the branch, so in this case the equilibrium solution is an exceptional case. The part of the branch of exponential solutions where $\lambda<0$ is biologically not very relevant, because these solutions are approximated by solutions of the nonhomogeneous system for a limited time interval only. We showed in Subsect. 4.4 that the bifurcation of the branch of exponential solutions is supercritical for values of $\xi$ above the threshold value $\xi_{T}<1$ and subcritical for $\xi<\xi_{T}$.

\section{Conclusions}

The interaction of disease transmission and population growth takes subtle forms and may produce complicated patterns of dynamical behaviour. Key ingredients of any model are the influence of the disease on the mortality and the fertility of the hosts and the way in which the force of infection depends on 
population size and composition. We have incorporated a saturating force of infection as well as additional mortality and reduced fertility due to the disease.

For a contact parameter $\kappa$ (the least upper bound for the force of infection) a number of critical values were established. The intuitive idea that the disease gets a stronger hold on the population when $\kappa$ is increased, is substantiated, but needs a careful interpretation in view of the possibility of bistable behaviour. The latter means that, for given parameter values, the disease may or may not reduce the growth rate of the population (or even regulate the population), depending on the initial conditions.

We have shown in the foregoing analysis for two types of epidemic models that incorporating a "multiplicative" negative influence of the disease on the fertility of the infectives can lead to sustained (and even large) oscillations in the sizes of the populations under consideration. This phenomenon does not occur, if the influence on fertility is modelled additively, as was done in [16] for the host-parasite model. For model I it can be shown that it is essential that we take the pair formation between infectives and susceptibles into account. Indeed, if the birth term in (2.1) is described by $\beta(S+\xi I)$, the equations do not have periodic solutions. (It was brought to our attention by Professor R. M. May [15] that this form arises as well when we do incorporate pair formation, but assume that reduced fertility is a consequence of vertical transmission followed by the death of the offspring before reaching the reproductive age, where only mothers can transmit the disease vertically.) This means that the interaction between infectives and susceptibles is important, i.e. the way in which an infective individual in effect reduces the fertility of the susceptible that it is paired with.

The phenomenon of oscillating solutions in model I is interesting, because up to now oscillations in epidemic models of the $S-I$ or $S-I-R$ type were only found for models, which contained time delays or periodic forcing terms (see for instance [10]). In 1981 Hethcote et al. [11] were led to the conjecture that "a constant-parameter model for a single, homogeneously mixing, uniform population can have periodic solutions for some parameter values if and only if the model is cyclic and involves temporary immunity through which individuals can be significantly delayed in the immune class". As far as we know model I is the first model which contradicts this conjecture.

It is now an open question whether oscillatory behaviour in real biological host-parasite systems may be ascribed to the influence of the parasite on the fertility of the host.

We hope that the analysis of this paper has contributed to the knowledge of possible relations between mechanisms and resulting phenomena.

\section{Appendix}

In order to apply the general results for (4.5) to the specific examples (4.2) and (4.4) one has to determine $\bar{y}, g$ and $y_{\max }$ and from these ingredients one then can calculate $\kappa_{1}, \kappa_{2}, \kappa_{T}$ and $\xi_{T}$. We shall now carry out this program and meanwhile also check the various assumptions.

Model $I$. We use the notation $\eta=1-\xi$. From

$$
F(y)=\mu+\alpha y-\beta(1-\eta y)^{2}
$$


we deduce

$$
F^{\prime}(y)=\alpha+2 \beta \eta(1-\eta y)>0
$$

for $0 \leqslant y<1$ and $0 \leqslant \eta \leqslant 1$. Furthermore III(i):

$$
\frac{\partial F}{\partial \eta}=2 \beta y(1-\eta y)>0
$$

Solving the quadratic equation $F(y)=0$ we find

$$
\bar{y}=\frac{1}{2 \eta^{2}}\left(2 \eta+\frac{\alpha}{\beta}-\sqrt{\left(2 \eta+\frac{\alpha}{\beta}\right)^{2}-4 \eta^{2}\left(1-\frac{\mu}{\beta}\right)}\right)
$$

(we have to take the negative square root to ensure $\bar{y} \in(0,1)$ ), which yields $I(i)$.

From

$$
G(\kappa, x, y)=\left(\frac{\kappa}{c x+1}-\alpha\right)(1-y)-\beta(1-\eta y)^{2}
$$

we conclude that

$$
g(\kappa, y)=-\frac{1}{c}+\frac{\kappa(1-y)}{c\left(\alpha(1-y)+\beta(1-\eta y)^{2}\right)} .
$$

Since

(i) $G_{y}(\kappa, g(\kappa, y), y)=-\beta \frac{(1-\eta y)^{2}}{1-y}+2 \beta \eta(1-\eta y)$

is independent of $\kappa$,

(ii) $g_{y}(\kappa, y)=\frac{\kappa \beta(1-\eta y)(\eta(2-y)-1)}{c\left(\alpha(1-y)+\beta(1-\eta y)^{2}\right)^{2}}$

equals zero for $y \in[0,1) \Leftrightarrow\left(y=y_{\max }:=2-(1 / \eta)\right.$ and $\left.\eta>\frac{1}{2}=\eta_{T}\right)$

(iii) $\lim _{y \rightarrow 1} g(\kappa, y)=\left\{\begin{array}{l}-\frac{1}{c} \text { for } \eta<1 \\ \frac{1}{c}\left(-1+\frac{\kappa}{\alpha}\right) \text { for } \eta=1\end{array}\right.$

we see that Assumptions II hold for $\eta<1$, but that the case $\eta=1$ deserves special attention (see below). Assumptions III(ii) and (iii) follow directly from the explicit expression for $y_{\max }$.

The equation $F(0)=G(\kappa, 0,0)$ amounts to $\mu-\beta=\kappa-\alpha-\beta$ from which we can conclude that $\kappa_{0}=\mu+\alpha$.

The equation $g=0$ reduces to a quadratic equation whose solutions are

$$
y^{*}=\frac{1}{2 \eta^{2}}\left(2 \eta+\frac{\alpha-\kappa}{\beta} \pm \sqrt{\left(2 \eta+\frac{\alpha-\kappa}{\beta}\right)^{2}-4 \eta^{2}\left(1-\frac{\kappa-\alpha}{\beta}\right)}\right)
$$

Putting $y^{*}=0$ amounts to requiring that $1-(\kappa-\alpha) / \beta=0$. Hence

$$
\kappa_{1}=\alpha+\beta \text {. }
$$

For $\kappa>\kappa_{1}$ only the " + " sign yields a solution in $(0,1)$. 

find that

The threshold value $\kappa_{T}$ should be such that $g\left(\kappa, y_{\max }\right)=0$ from which we

$$
\kappa_{T}=\alpha+4 \beta \eta(1-\eta)
$$

provided $\eta>\eta_{T}=\frac{1}{2}$, (i.e. $\xi_{T}=\frac{1}{2}$ ). Note that $\kappa_{T} \rightarrow \alpha$ for $\eta \rightarrow 1$, so that $\kappa_{T}$ can even be smaller than $\kappa_{0}$.

The condition $\bar{y}=y^{*}$ amounts to

$$
\frac{\kappa}{\beta}-\sqrt{\left(2 \eta+\frac{\alpha}{\beta}\right)^{2}-4 \eta^{2}\left(1-\frac{\mu}{\beta}\right)}= \pm \sqrt{\left(2 \eta+\frac{\alpha-\kappa}{\beta}\right)^{2}-4 \eta^{2}\left(1-\frac{\kappa-\alpha}{\beta}\right)}
$$

Squaring both sides and solving for $\kappa$ we find

$$
\kappa_{2}=\frac{2(\alpha+\mu) \eta^{2}}{2 \eta^{2}-\left(2 \eta+\frac{\alpha}{\beta}\right)+\sqrt{\left(2 \eta+\frac{\alpha}{\beta}\right)^{2}-4 \eta^{2}\left(1-\frac{\mu}{\beta}\right)}} .
$$

For $\eta \rightarrow 0$ we find

$$
\kappa_{2} \rightarrow \frac{\alpha(\mu+\alpha)}{\mu+\alpha-\beta} .
$$

The critical value $\eta_{p}$ can be equivalently characterized by $y_{\max }=\bar{y}$ or $\kappa_{2}=\kappa_{T}$. Elaborating one of these we find the cubic equation

$$
4 \beta \eta^{3}-8 \beta \eta^{2}+(4 \beta-2 \alpha-\mu) \eta+\alpha=0 .
$$

The left hand side is positive for $\eta=\frac{1}{2}$ and negative for $\eta=1$, so in $\left(\frac{1}{2}, 1\right)$ there exists a unique root $\eta_{p}$.

Finally, we look at the special case $\eta=1$ (i.e. $\xi=0$ ). It is now more natural to include $y=1$ in the set $M$, since the system

$$
\begin{aligned}
& \frac{d x}{d t}=x\left(\mu+\alpha y-\beta(1-y)^{2}\right) \\
& \frac{d y}{d t}=y(1-y)\left(\frac{\kappa}{c x+1}-\alpha-\beta(1-y)\right)
\end{aligned}
$$

has $(x, y)=(0,1)$ as a steady state. The bifurcation diagram for this situation is shown in Fig. 13.

Drawing phase portraits we arrive at the conclusion that necessarily $\kappa_{p}=\kappa_{1}$. More precisely, the limit cycle bifurcates at $\kappa=\kappa_{1}$ from the "degenerate" saddle

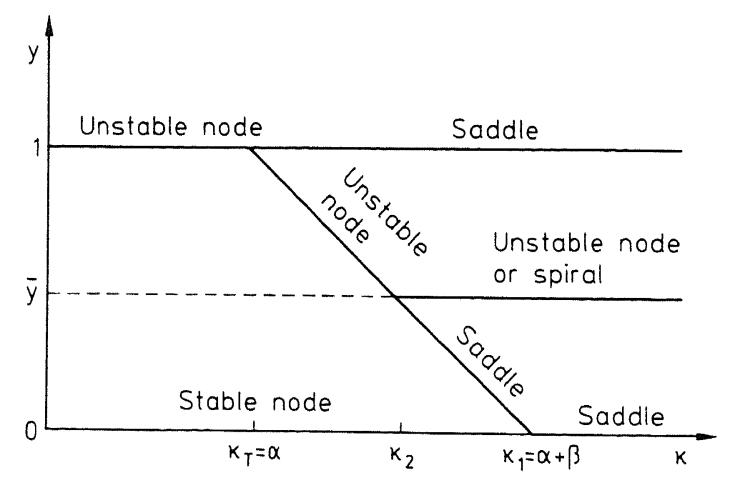

Fig. 13 
connection given by the trajectory on the $y$-axis connecting $(0,0)$ and $(0,1)$, the unstable manifold of $(0,1)$ (the straight line $y=1$ ), which at infinity connects to the stable manifold of $(0,0)$ (the $x$-axis). This result illustrates that the periodic outbreaks for $\kappa$ slightly larger than $\kappa_{1}$ are indeed huge.

Dr. Jin Cheng-Fu [12] informs us that he can prove the uniqueness of the limit cycle for $\xi \ll 1$ by using a modified version of Zhang's theorem (see [14]).

Model II. From

we deduce I(i):

$$
F(y)=\alpha y+\mu-\beta\left(\frac{k}{(1-\xi) y+k}\right)^{k}
$$

Furthermore we see that

$$
F^{\prime}(y)=\alpha+\beta(1-\xi)\left(\frac{k}{(1-\xi) y+k}\right)^{k+1}>0 .
$$

$$
F(y)=0 \Leftrightarrow \alpha y+\mu=\beta\left(\frac{k}{(1-\xi) y+k}\right)^{k} .
$$

The left hand side of the second equation is a straight line with slope $\alpha$, the right hand side a hyperbola which intersects the $(y=0)$-axis at $\beta$ and goes to zero for $y \rightarrow \infty$. This shows that there exists precisely one $\bar{y}>0$ with $F(\bar{y})=0$ provided $\mu<\beta$. In general $\bar{y}$ cannot be calculated explicitly. From

we get I(ii)

$$
G(\kappa, x, y)=\frac{\kappa}{c x+1}-(\sigma+\alpha)-\frac{\alpha}{k} y-\beta\left(\frac{k}{(1-\xi) y+k}\right)^{k}
$$

and I(iii)

$$
G_{x}(\kappa, x, y)=-\frac{c \kappa}{(c x+1)^{2}}<0
$$

$$
\begin{aligned}
G_{k}(\kappa, x, y) & =\frac{1}{c x+1}>0, \\
G(0, x, y) & =-(\sigma+\alpha)-\frac{\alpha}{k} y-\beta\left(\frac{k}{(1-\xi) y+k}\right)^{k}<0 .
\end{aligned}
$$

Furthermore we see that

$$
G_{y}(\kappa, x, y)=-\frac{\alpha}{k}+\beta(1-\xi)\left(\frac{k}{(1-\xi) y+k}\right)^{k+1}
$$

is independent of $\kappa$, which ensures II(i). We calculate $g(\kappa, y)$ from $G(\kappa, x, y)=0$ as

$$
g(\kappa, y)=\frac{\kappa}{c\left(\sigma+\alpha+\frac{\alpha}{k} y+\beta\left(\frac{k}{(1-\xi) y+k}\right)^{k}\right)}-\frac{1}{c} .
$$

To study $g$ we find the derivative with respect to $y$ as

$$
g_{y}(\kappa, y)=\frac{\kappa}{c} \frac{\left(\beta(1-\xi)\left(\frac{k}{(1-\xi) y+k}\right)^{k+1}-\frac{\alpha}{k}\right)}{\left(\sigma+\alpha+\frac{\alpha}{k} y+\beta\left(\frac{k}{(1-\xi) y+k}\right)^{k}\right)^{2}} .
$$


So we get

$$
g_{y}(\kappa, y)=0 \Leftrightarrow \frac{\alpha}{k}=\beta(1-\xi)\left(\frac{k}{(1-\xi) y+k}\right)^{k+1} .
$$

Again the right hand side of the second equation is a hyperbola which intersects the $(y=0)$-axis at $\beta(1-\xi)$ and tends to zero for $y \rightarrow \infty$, while the left hand side is a parallel to the $y$-axis at $\alpha / k$. So again there exists precisely one $y=y_{\max }$ for which $g_{y}\left(\kappa, y_{\max }\right)=0$ provided $\beta(1-\xi)>\alpha / k$, which is equivalent to $\xi<\xi_{T}$. (The threshold $\xi_{T}$ was already computed in Subsect. 4.4.) That $y_{\max }$ is indeed a local maximum can be seen by evaluating the second derivative with respect to $y$ of $g$ at $y_{\max }$

$$
g_{y y}\left(\kappa, y_{\max }\right)=-\frac{\kappa}{c} \frac{\beta(1-\xi)^{2}\left(\frac{k+1}{k}\right)\left(\frac{k}{(1-\xi) y_{\max }+k}\right)^{k+1}}{\left(\sigma+\alpha+\frac{\alpha}{k} y_{\max }+\beta\left(\frac{k}{(1-\xi) y_{\max }+k}\right)^{k}\right)^{2}}<0 .
$$

Also condition II(iii) is fulfilled, because

$$
\lim _{y \rightarrow \infty}=-\frac{1}{c}
$$

Now we turn to the conditions concerning $\xi$. First of all we get

$$
\frac{\partial F}{\partial \xi}=-\beta y\left(\frac{k}{(1-\xi) y+k}\right)^{k+1}<0,
$$

which is condition III(i). To prove III(ii) we consider a function $f$ which implicitly defines $y_{\max }$ as a function of $\xi$ :

$$
f\left(y_{\max }, \xi\right)=\beta(1-\xi)\left(\frac{k}{(1-\kappa) y_{\max }+k}\right)^{k+1}-\frac{\alpha}{k}=0 .
$$

By the Implicit Function Theorem we can now write

$$
\frac{d y_{\max }}{d \xi}=-\frac{\partial f}{\partial \xi} / \frac{\partial f}{\partial y_{\max }}
$$

provided the partial derivative of $f$ with respect to $y_{\max }$ is not zero. We compute

$$
\frac{\partial f}{\partial \xi}=\beta\left(\frac{k}{(1-\xi) y_{\max }+k}\right)^{k+1}\left(\frac{(1-\xi)(k+1) y_{\max }}{(1-\xi) y_{\max }+k}-1\right)
$$

and

$$
\frac{\partial f}{\partial y_{\max }}=-\beta(1-\xi)^{2} \frac{k+1}{k}\left(\frac{k}{(1-\xi) y_{\max }+k}\right)^{k+2}<0 .
$$

This leads to

$$
\frac{d y_{\max }}{d \xi}=\frac{k\left(1-(1-\xi) y_{\max }\right)}{(k+1)(1-\xi)^{2}}
$$

So we get the condition

$$
\frac{d y_{\max }}{d \xi}<0 \Leftrightarrow y_{\max }>\frac{1}{1-\xi} .
$$


Recalling the definition of $y_{\max }$ we see that this is equivalent to

$$
\beta(1-\xi)\left(\frac{k}{1+k}\right)^{k+1}>\frac{\alpha}{k} \text {. }
$$

The inequality is valid for all $k \geqslant 0$ provided $\beta(1-\xi)>\alpha / k$, i.e. $\xi<\xi_{T}$.

To prove condition III(iii) we consider $y_{\max }$ which is in the case $\xi=0$ characterized by

We solve for $y_{\max }$ and get

$$
\frac{\alpha}{k}=\beta\left(\frac{k}{y_{\max }+k}\right)^{k+1} .
$$

$$
y_{\max }=k\left(\sqrt[k+1]{\frac{k \beta}{\alpha}}-1\right) .
$$

Now $y_{\max }>\bar{y}$ amounts to

So with

$$
\frac{\alpha}{\beta k}<\left(\frac{\bar{y}}{k}+1\right)^{-(k+1)}
$$

$$
\varepsilon:=\left(\frac{\bar{y}}{k}+1\right)^{-(k+1)}<1
$$

we get that III(iii) is valid, if $\alpha / \beta k<\varepsilon$.

Now we calculate the thresholds for $\kappa$.

From $F(0)=G\left(\kappa_{0}, 0,0\right)$ we see immediately that

$$
\kappa_{0}=\mu+\sigma+\alpha \text {. }
$$

From $G\left(\kappa_{1}, 0,0\right)=0$ we get

$$
\kappa_{1}=\beta+\sigma+\alpha
$$

The threshold $\kappa_{2}$ is characterized by $g\left(\kappa_{2}, \bar{y}\right)=0$. As $\bar{y}$ is characterized by

$$
\alpha \bar{y}+\mu=\beta\left(\frac{k}{(1-\xi) \bar{y}+k}\right)^{k}
$$

we can write

which implies

$$
g\left(\kappa_{2}, \bar{y}\right)=\frac{\kappa_{2}}{c\left(\sigma+\alpha+\mu+\alpha\left(\frac{1+k}{k}\right) \bar{y}\right)}-\frac{1}{c}=0,
$$

$$
\kappa_{2}=\sigma+\alpha+\mu+\alpha\left(\frac{1+k}{k}\right) \bar{y} .
$$

In Subsect. 4.4 we formulated $\kappa$ as a function of $y$ along the bifurcation branch.

A value $y=\theta$ is characterized by $\kappa^{\prime}(\theta)=0$, or, equivalently, by

$$
\frac{\alpha}{k}=\beta(1-\xi)\left(\frac{k}{(1-\xi) \theta+k}\right)^{k+1} \text {. }
$$

We solve for $\theta$ and get

$$
\theta=\frac{k}{1-\xi}\left(\sqrt[k+1]{\frac{k \beta(1-\xi)}{\alpha}}-1\right) .
$$


Now $\kappa_{T}$ is given by $\kappa_{T}=\kappa(\theta)$ :

$$
\kappa(\theta)=\sigma+\alpha+\frac{\alpha}{1-\xi}\left(\sqrt[k+1]{\frac{k \beta(1-\xi)}{\alpha}}-1\right)+\beta\left(\sqrt[k+1]{\frac{\alpha}{k \beta(1-\xi)}}\right)^{k} .
$$

The threshold $\xi_{T}$ was already computed in Subsect. 4.4. The threshold $\xi_{p}$ cannot be given explicitly.

\section{References}

1. Anderson, R. M., May, R. M.: Regulation and stability of host-parasite population interactions. I. Regulatory processes, J. Anim. Ecol. 47, 219-247 (1978)

2. Anderson, R. M., May, R. M.: Population biology of infectious diseases: Part I. Nature 280, $361-367$ (1979)

3. Andreasen, V.: Disease regulation of age-structured host populations. Theor. Pop. Biol. 36, 214-239 (1989)

4. Blythe, S. P., Castillo-Chavez C.: Like-with-like preference and sexual mixing models, Math. Biosci. 96, 221-238 (1989)

5. Busenberg, S., Van den Driesche P.: Analysis of a disease transmission model in a population with varying size J. Math. Biol. 28, 257-270 (1990)

6. Dietz, K.: Overall population patterns in the transmission cycle of infectious disease agents. In: Anderson, R. M., May R. M. (eds.) Population Biology of Infectious Diseases. (Report of Dahlem Workshop, pp. 87-102) Berlin Heidelberg New York: Springer 1982

7. Dietz, K., Hadeler, K. P.: Epidemiological models for sexually transmitted diseases. J. Math. Biol. 26, 1-25 (1988)

8. Hadeler, K. P., Dietz, K.: Nonlinear hyperbolic partial differential equations for the dynamics of parasite populations. Comput. Math. Appl. 9, 415-430 (1983)

9. Hadeler, K. P., Dietz, K.: Population dynamics of killing parasites which reproduce in the host. J. Math. Biol. 21, 45-65 (1984)

10. Hethcote, H. W., Stech, H. W., Van den Driesche, P.: Periodicity and stability in epidemic models: A survey. In: S. N. Busenberg, K. I. Cooke (eds.) Differential equations and applications in ecology, epidemics, and population problems, pp. 65-82. New York: Academic Press 1981

11. Hethcote, H. W., Stech H. W., Van den Driesche, P.: Nonlinear oscillations in epidemic models. SIAM J. Appl. Math. 40, 1-9 (1981)

12. Jin Cheng-Fu: personal communication

13. Kretzschmar, M.: Persistent solutions in a model for parasitic diseases. J. Math. Biol. 27, 549-573 (1989)

14. Kuang Y., Freedman, H. I.: Uniqueness of limit cycles in Gause-type models of predator-prey systems. Math. Biosci. 88, 67-84 (1988)

15. May, R. M.: personal communication

16. May, R. M., Anderson, R. M.: Regulation and stability of host-parasite population interactions. II. Destabilizing processes. J. Anim. Ecol. 47, 249-267 (1978)

17. May, R. M., Anderson, R. M.: Population biology of infectious diseases: Part II. Nature 280, 455-461 (1979)

18. Waldstätter, R.: Pair formation in sexually transmitted diseases. In: C. Castillo-Chavez (ed.) Mathematical and statistical approaches to AIDS epidemiology. (Lect. Notes Biomath., vol. 83) Berlin Heidelberg New York: Springer 1989

19. Ye, Yan-Qian: Theory of limit cycles. Translation of Mathematical Monographs, Vol. 66. AMS, Providence, R.I., 1986

\section{Reference added in proof}

Busenberg, S., Cooke, K. L., Thieme, H. R.: Demographic change and persistence of HIV/AIDS in a heterogeneous population. SIAM J. Appl. Math. (to appear) 